\title{
Measurement of Coupling Resonance Driving Terms with the AC Dipole
}

\author{
R. Miyamoto
}

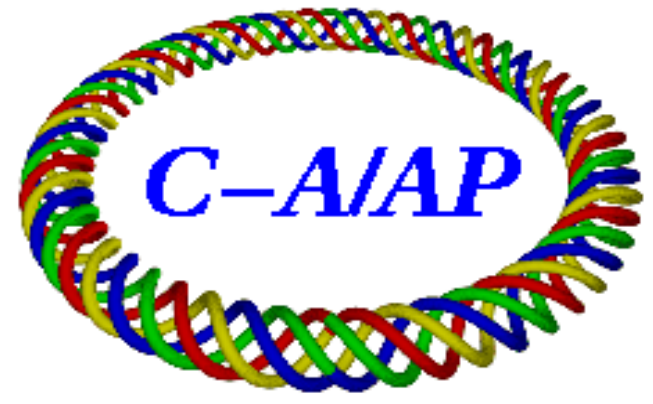

\section{Collider-Accelerator Department Brookhaven National Laboratory \\ Upton, NY 11973}

Notice: This document has been authorized No. DE-AC02-98CH10886 with the U.S. Department of Energy. The United States Government retains a non- 


\title{
Measurement of Coupling Resonance Driving Terms with the AC Dipole
}

\author{
Ryoichi Miyamoto
}

October 27, 2010

\begin{abstract}
Resonance driving terms for linear coupled betatron motion in a synchrotron ring can be determined from corresponding spectral lines of an excited coherent beam motion. An AC dipole is one of instruments to excite such a motion. When a coherent motion is excited with an AC dipole, measured Courant-Snyder parameters and betatron phase advance have apparent modulations, as if there is an additional quadrupole field at the location of the AC dipole. Hence, measurements of these parameters using the AC dipole require a proper interpretation of observed quantities. The situation is similar in measurements of resonance driving terms using the AC dipole. In this note, we derive an expression of coupled betatron motion excited with two AC dipoles in presence of skew quadrupole fields, discuss an impact of this quadrupole like effect of the $\mathrm{AC}$ dipole on a measurement of coupling resonance driving terms, and present an analytical method to determine the coupling resonance driving terms from quantities observed using the AC dipole.
\end{abstract}

\section{Introduction}

An $\mathrm{AC}$ dipole is one of instruments, such as a kicker dipole magnet, to excite a coherent beam motion in a synchrotron ring [1]. Observations of the excited coherent beam motion with beam position monitors (BPMs) allow prompt measurements of beam optical parameters in the ring. Optical parameters which can be measured in this way are not only standard Courant-Snyder parameters and (betatron) phase advance. Resonance driving terms due perturbative fields can be also measured from spectra of the coherent beam motion [2].

The motion excited with an AC dipole, referred to as "driven (betatron) motion", is not completely identical to the normal betatron motion, referred to as "free (betatron) motion" in contrast to the driven motion, in the same ring [3, 4]. The difference can be interpreted as an additional quadrupole field at the location of the AC dipole and the driven motion is parametrized with a new set of Courant-Snyder parameters and phase advance [5]. In presence of the AC dipole, the modes driven by perturbative fields are also altered $[6,7]$. In this note, we derive an analytical expression of coupled driven motion excited with two AC dipoles in presence of weak skew quadrupole fields and demonstrate that the motion can be parametrized with a new set of coupling resonance driving terms (CRDTs), similar to the case of the one-dimensional driven motion. We also present an analytical procedure to determine the normal CRDTs for the free motion from the new CRDTs for the driven motion, which are the quantities directly measured by observing the driven motion.

\section{Review of Basic Concepts}

In this section, we review a few basic concepts and establish notations used in this note.

\subsection{Complex Representation of Phase Space}

In this section, we review a complex representation of phase space used in this note. Equations of motion for twodimensional betatron motion are given by

$$
\begin{aligned}
& \frac{d^{2} x}{d s^{2}}+K_{x}(s) x=-\frac{\Delta B_{y}(x, y, s)}{(B \rho)} \\
& \frac{d^{2} y}{d s^{2}}+K_{y}(s) y=\frac{\Delta B_{x}(x, y, s)}{(B \rho)},
\end{aligned}
$$


where $s(-\infty<s<\infty)$ is longitudinal coordinate, $K_{x}(s)$ and $K_{y}(s)$ are effective gradients, $\Delta B_{x}(x, y, s)$ and $\Delta B_{y}(x, y, s)$ are perturbative magnetic fields, and $(B \rho)$ is magnetic rigidity. When we consider turn-by-turn motion of a beam observed at a fixed location in a synchrotron ring, it may be convenient to rewrite the longitudinal coordinate $s$ with number of revolution $n$, circumference of the ring $C$, and a parameter $\bar{s}(0 \leq \bar{s}<C)$ denoting the location of the observation:

$$
s=n C+\bar{s} .
$$

Mathematically, $n$ is the largest integer smaller than or equal to $s / C$ and $\bar{s}$ is the reminder of $s / C$. By using $n$ and $\bar{s}$, the solutions of Equations (1) and (2) are occasionally written as $x(n ; \bar{s})$ and $y(n ; \bar{s})$ instead of $x(s)$ and $y(s)$ in this note. A periodic function of $s$ such as $K_{x}(s)$ obviously satisfies $K_{x}(s)=K_{x}(\bar{s})$. The well known solutions of the homogeneous parts of Equations (1) and (2) are [8]

$$
\begin{aligned}
& x(n ; \bar{s})=A_{x} \sqrt{\beta_{x}(\bar{s})} \cos \left[2 \pi \nu_{x} n+\psi_{x}(\bar{s})+\phi_{x}\right] \\
& y(n ; \bar{s})=A_{y} \sqrt{\beta_{y}(\bar{s})} \cos \left[2 \pi \nu_{y} n+\psi_{y}(\bar{s})+\phi_{y}\right]
\end{aligned}
$$

and their slopes are

$$
\begin{aligned}
& x^{\prime}(n ; \bar{s})=-\frac{A_{x}}{\sqrt{\beta_{x}(\bar{s})}} \sin \left[2 \pi \nu_{x} n+\psi_{x}(\bar{s})+\phi_{x}\right]-\frac{A_{x} \alpha_{x}(\bar{s})}{\sqrt{\beta_{x}(\bar{s})}} \cos \left[2 \pi \nu_{x} n+\psi_{x}(\bar{s})+\phi_{x}\right] \\
& y^{\prime}(n ; \bar{s})=-\frac{A_{y}}{\sqrt{\beta_{y}(\bar{s})}} \sin \left[2 \pi \nu_{x} n+\psi_{y}(\bar{s})+\phi_{y}\right]-\frac{A_{y} \alpha_{y}(\bar{s})}{\sqrt{\beta_{y}(\bar{s})}} \cos \left[2 \pi \nu_{x} n+\psi_{y}(\bar{s})+\phi_{y}\right],
\end{aligned}
$$

where the prime denotes derivative with respect to $s, A_{x}, A_{y}, \phi_{x}$, and $\phi_{y}$ are constants of motion, $\beta_{x}(\bar{s}), \beta_{y}(\bar{s}), \alpha_{x}(\bar{s})$, and $\alpha_{y}(\bar{s})$ are Courant-Snyder parameters, $\psi_{x}(s)$ and $\psi_{y}(s)$ are phase advances from $s=0$ to $s$, and $\nu_{x}=\psi_{x}(C) / 2 \pi$ and $\nu_{y}=\psi_{y}(C) / 2 \pi$ are betatron tunes. The phase advances from an arbitrary point $s_{1}$ to the other $s_{2}$ are denoted by $\psi_{x}\left(s_{2}, s_{1}\right)$ and $\psi_{y}\left(s_{2}, s_{1}\right)$. The phase space positions of these solutions can be expressed in compact forms in complex representations $\tilde{x}=x+i\left[\alpha_{x}(\bar{s}) x+\beta_{x}(\bar{s}) x^{\prime}\right]$ and $\tilde{y}=y+i\left[\alpha_{y}(\bar{s}) y+\beta_{y}(\bar{s}) y^{\prime}\right]$ :

$$
\begin{aligned}
& \tilde{x}(n ; \bar{s})=A_{x} \sqrt{\beta_{x}(\bar{s})} e^{-2 \pi i \nu_{x} n-i \psi_{x}(\bar{s})-i \phi_{x}} \\
& \tilde{y}(n ; \bar{s})=A_{y} \sqrt{\beta_{y}(\bar{s})} e^{-2 \pi i \nu_{y} n-i \psi_{y}(\bar{s})-i \phi_{y}},
\end{aligned}
$$

We use symbols $\mathscr{X}(\nu ; \bar{s})$ and $\mathscr{Y}(\nu ; \bar{s})$ for Fourier components of $\tilde{x}(n ; \bar{s})$ and $\tilde{y}(n ; \bar{s})$ with tune $\nu$. We can choose the normalization of the Fourier Transformation so that

$$
\begin{aligned}
\mathscr{X}\left(-\nu_{x} ; \bar{s}\right) & =A_{x} \sqrt{\beta_{x}(\bar{s})} e^{-i \psi_{x}(\bar{s})-i \phi_{x}} \\
\mathscr{Y}\left(-\nu_{y} ; \bar{s}\right) & =A_{y} \sqrt{\beta_{y}(\bar{s})} e^{-i \psi_{y}(\bar{s})-i \phi_{y}} .
\end{aligned}
$$

Because BPMs can measure only positions, $x(n ; \bar{s})$ and $y(n ; \bar{s})$, and not slopes, $x^{\prime}(n ; \bar{s})$ and $y^{\prime}(n ; \bar{s})$, we have to use two adjacent BPMs to construct phase space at one BPM location [9]. We consider two adjacent BPMs located at $\bar{s}_{1}$ and $\bar{s}_{2}$. From Equations (4), (5), (6), (7), (8), and (9) the following equalities hold:

$$
\begin{aligned}
& \tilde{x}\left(n ; \bar{s}_{1}\right)=\frac{1}{i \sin \left[\psi_{x}\left(\bar{s}_{2}, \bar{s}_{1}\right)\right]}\left[e^{i \psi_{x}\left(\bar{s}_{2}, \bar{s}_{1}\right)} x\left(n ; \bar{s}_{1}\right)-\sqrt{\left.\frac{\beta_{x}\left(\bar{s}_{1}\right)}{\beta_{x}\left(\bar{s}_{2}\right)} x\left(n ; \bar{s}_{2}\right)\right]}\right. \\
& \tilde{y}\left(n ; \bar{s}_{1}\right)=\frac{1}{i \sin \left[\psi_{y}\left(\bar{s}_{2}, \bar{s}_{1}\right)\right]}\left[e^{i \psi_{y}\left(\bar{s}_{2}, \bar{s}_{1}\right)} y\left(n ; \bar{s}_{1}\right)-\sqrt{\left.\frac{\beta_{y}\left(\bar{s}_{1}\right)}{\beta_{y}\left(\bar{s}_{2}\right)} y\left(n ; \bar{s}_{2}\right)\right] .}\right.
\end{aligned}
$$

We note that the phase advances $\psi_{x}\left(\bar{s}_{2}, \bar{s}_{1}\right)$ and $\psi_{y}\left(\bar{s}_{2}, \bar{s}_{1}\right)$ and the ratios of the $\beta$-functions $\beta_{x}\left(\bar{s}_{1}\right) / \beta_{x}\left(\bar{s}_{2}\right)$ and $\beta_{y}\left(\bar{s}_{1}\right) / \beta_{y}\left(\bar{s}_{2}\right)$ can be measured with these two BPMs. With these equations, the complex phase space position at the locations of BPMs can be constructed for every revolution.

\subsection{Uncoupled Driven Betatron Motion}

In this section, we review properties of the uncoupled driven motion excited by two orthogonal AC dipoles. When a perfectly uncoupled ring has one horizontal and one vertical AC dipoles, where the horizontal (vertical) AC dipole 
produces a vertical (horizontal) field and excites a horizontal (vertical) motion, the equations of motion are given by

$$
\begin{aligned}
& \frac{d^{2} x}{d s^{2}}+K_{x}(\bar{s}) x=-\sum_{m=-\infty}^{\infty} \theta_{h} \delta\left(s-\bar{s}_{h}-m C\right) \cos \left(2 \pi \nu_{x, h} m+\phi_{h}\right) \\
& \frac{d^{2} y}{d s^{2}}+K_{y}(\bar{s}) y=-\sum_{m=-\infty}^{\infty} \theta_{v} \delta\left(s-\bar{s}_{v}-m C\right) \cos \left(2 \pi \nu_{y, v} m+\phi_{v}\right)
\end{aligned}
$$

where $\delta(s)$ is Dirac's delta function and the subscripts " $h$ " or " $v$ " indicate that the parameter is related to the horizontal or vertical AC dipole: $\theta_{h}$ and $\theta_{v}$ are deflection angles of the AC dipoles, $\bar{s}_{h}$ and $\bar{s}_{v}\left(0 \leq \bar{s}_{h}, \bar{s}_{v}<C\right)$ are locations of the AC dipoles, $\nu_{x, h}$ and $\nu_{y, v}$ are driving tunes of the AC dipoles, and $\phi_{h}$ and $\phi_{v}$ are constant phases of the AC dipole fields. The minus signs on the right-hand-sides are a mere convention. When the deflection angles of the AC dipoles, $\theta_{h}$ and $\theta_{v}$, are adiabatic parameters, the particular solutions of these equations are given by $[3,4]$

$$
\begin{aligned}
x(n ; \bar{s})= & \frac{\theta_{h} \sqrt{\beta_{x}\left(\bar{s}_{h}\right)}}{4 \sin \left[\pi\left(\nu_{x, h}-\nu_{x}\right)\right]} \sqrt{\beta_{x}(\bar{s})} \cos \left[2 \pi \nu_{x, h} n+\psi_{x}\left(\bar{s}, \bar{s}_{h}\right)+\pi\left(\nu_{x, h}-\nu_{x}\right) \operatorname{sgn}\left(\bar{s}-\bar{s}_{h}\right)+\phi_{h}\right] \\
& -\frac{\theta_{h} \sqrt{\beta_{x}\left(\bar{s}_{h}\right)}}{4 \sin \left[\pi\left(\nu_{x, h}+\nu_{x}\right)\right]} \sqrt{\beta_{x}(\bar{s})} \cos \left[2 \pi \nu_{x, h} n-\psi_{x}\left(\bar{s}, \bar{s}_{h}\right)+\pi\left(\nu_{x, h}+\nu_{x}\right) \operatorname{sgn}\left(\bar{s}-\bar{s}_{h}\right)+\phi_{h}\right]
\end{aligned}
$$

and

$$
\begin{aligned}
y(n ; \bar{s})= & \frac{\theta_{v} \sqrt{\beta_{y}\left(\bar{s}_{v}\right)}}{4 \sin \left[\pi\left(\nu_{y, v}-\nu_{y}\right)\right]} \sqrt{\beta_{y}(\bar{s})} \cos \left[2 \pi \nu_{y, v} n+\psi_{y}\left(\bar{s}, \bar{s}_{v}\right)+\pi\left(\nu_{y, v}-\nu_{y}\right) \operatorname{sgn}\left(\bar{s}-\bar{s}_{v}\right)+\phi_{v}\right] \\
& -\frac{\theta_{v} \sqrt{\beta_{y}\left(\bar{s}_{v}\right)}}{4 \sin \left[\pi\left(\nu_{y, v}+\nu_{y}\right)\right]} \sqrt{\beta_{y}(\bar{s})} \cos \left[2 \pi \nu_{y, v} n-\psi_{y}\left(\bar{s}, \bar{s}_{v}\right)+\pi\left(\nu_{y, v}+\nu_{y}\right) \operatorname{sgn}\left(\bar{s}-\bar{s}_{v}\right)+\phi_{v}\right]
\end{aligned}
$$

where $\operatorname{sgn}(s)=s /|s|$ is sign function. In the complex representation discussed in the previous section, the solutions are given by

$$
\begin{aligned}
\tilde{x}(n ; \bar{s})= & \frac{\theta_{h} \sqrt{\beta_{x}\left(\bar{s}_{h}\right)}}{4 \sin \left[\pi\left(\nu_{x, h}-\nu_{x}\right)\right]} \sqrt{\beta_{x}(\bar{s})} e^{-2 \pi i \nu_{x, h} n-i \psi_{x}\left(\bar{s}, \bar{s}_{h}\right)-\pi i\left(\nu_{x, h}-\nu_{x}\right) \operatorname{sgn}\left(\bar{s}-\bar{s}_{h}\right)-i \phi_{h}} \\
& -\frac{\theta_{h} \sqrt{\beta_{x}\left(\bar{s}_{h}\right)}}{4 \sin \left[\pi\left(\nu_{x, h}+\nu_{x}\right)\right]} \sqrt{\beta_{x}(\bar{s})} e^{2 \pi i \nu_{x, h} n-i \psi_{x}\left(\bar{s}, \bar{s}_{h}\right)+\pi i\left(\nu_{x, h}+\nu_{x}\right) \operatorname{sgn}\left(\bar{s}-\bar{s}_{h}\right)+i \phi_{h}}
\end{aligned}
$$

and

$$
\begin{aligned}
\tilde{y}(n ; \bar{s})= & \frac{\theta_{v} \sqrt{\beta_{y}\left(\bar{s}_{v}\right)}}{4 \sin \left[\pi\left(\nu_{y, v}-\nu_{y}\right)\right]} \sqrt{\beta_{y}(\bar{s})} e^{-2 \pi i \nu_{y, v} n-i \psi_{y}\left(\bar{s}, \bar{s}_{v}\right)-\pi i\left(\nu_{y, v}-\nu_{y}\right) \operatorname{sgn}\left(\bar{s}-\bar{s}_{v}\right)-i \phi_{v}} \\
& -\frac{\theta_{v} \sqrt{\beta_{y}\left(\bar{s}_{v}\right)}}{4 \sin \left[\pi\left(\nu_{y, v}+\nu_{y}\right)\right]} \sqrt{\beta_{y}(\bar{s})} e^{2 \pi i \nu_{y, v} n-i \psi_{y}\left(\bar{s}, \bar{s}_{v}\right)+\pi i\left(\nu_{y, v}+\nu_{y}\right) \operatorname{sgn}\left(\bar{s}-\bar{s}_{v}\right)+i \phi_{v}} .
\end{aligned}
$$

The two terms in Equations (16), (17), (18), and (19) represent modes of difference and sum resonances driven by one $\mathrm{AC}$ dipole. We note that the modes of the difference and sum resonances rotate in opposite directions in the phase space.

Equations (16) and (17) can be written in simpler forms if we introduce new optical parameters for the driven motion, $\beta_{x, h}(\bar{s}), \beta_{y, v}(\bar{s}), \alpha_{x, h}(\bar{s}), \alpha_{y, v}(\bar{s}), \psi_{x, h}\left(s_{2}, s_{1}\right)$, and $\psi_{y, v}\left(s_{2}, s_{1}\right),[5]$ :

$$
\begin{aligned}
& x(n ; \bar{s})=A_{x, h} \sqrt{\beta_{x, h}(\bar{s})} \cos \left[2 \pi \nu_{x, h} n+\psi_{x, h}\left(\bar{s}, \bar{s}_{h}\right)+\phi_{h}\right] \\
& y(n ; \bar{s})=A_{y, v} \sqrt{\beta_{y, v}(\bar{s})} \cos \left[2 \pi \nu_{y, v} n+\psi_{y, v}\left(\bar{s}, \bar{s}_{v}\right)+\phi_{v}\right]
\end{aligned}
$$

and, then, the slopes are given by

$$
\begin{aligned}
& x^{\prime}(n ; \bar{s})=-\frac{A_{x, h}}{\sqrt{\beta_{x, h}(\bar{s})}} \sin \left[2 \pi \nu_{x, h} n+\psi_{x, h}\left(\bar{s}, \bar{s}_{h}\right)+\phi_{h}\right]-\frac{A_{x, h} \alpha_{x, h}(\bar{s})}{\sqrt{\beta_{x, h}(\bar{s})}} \cos \left[2 \pi \nu_{x, h} n+\psi_{x, h}\left(\bar{s}, \bar{s}_{h}\right)+\phi_{h}\right] \\
& y^{\prime}(n ; \bar{s})=-\frac{A_{y, v}}{\sqrt{\beta_{y, v}(\bar{s})}} \sin \left[2 \pi \nu_{y, v} n+\psi_{y, v}\left(\bar{s}, \bar{s}_{v}\right)+\phi_{v}\right]-\frac{A_{y, v} \alpha_{y, v}(\bar{s})}{\sqrt{\beta_{y, v}(\bar{s})}} \cos \left[2 \pi \nu_{y, v} n+\psi_{y, v}\left(\bar{s}, \bar{s}_{v}\right)+\phi_{v}\right]
\end{aligned}
$$


where $A_{x, h}$ and $A_{y, v}$ are constants of motion:

$$
\begin{aligned}
& A_{x, h}=\frac{\theta_{h}}{4 \sin \left(\pi \delta_{h}\right)} \sqrt{\beta_{x}\left(\bar{s}_{h}\right)\left(1-\lambda_{h}^{2}\right)} \\
& A_{y, v}=\frac{\theta_{v}}{4 \sin \left(\pi \delta_{v}\right)} \sqrt{\beta_{y}\left(\bar{s}_{v}\right)\left(1-\lambda_{v}^{2}\right)},
\end{aligned}
$$

$\delta_{h}=\nu_{x, h}-\nu_{x}$ and $\delta_{v}=\nu_{y, v}-\nu_{y}$ are small parameters typically on the order of 0.01 or maybe less, and $\lambda_{h}$ and $\lambda_{v}$ are other small parameters describing the ratios of magnitudes of the difference and sum resonances:

$$
\begin{aligned}
& \lambda_{h}=\frac{\sin \left[\pi\left(\nu_{x, h}-\nu_{x}\right)\right]}{\sin \left[\pi\left(\nu_{x, h}+\nu_{x}\right)\right]} \\
& \lambda_{v}=\frac{\sin \left[\pi\left(\nu_{y, v}-\nu_{y}\right)\right]}{\sin \left[\pi\left(\nu_{y, v}+\nu_{y}\right)\right]} .
\end{aligned}
$$

The new optical parameters of the driven motion are related to the corresponding parameters of the free motion by

$$
\begin{aligned}
\beta_{x, h}(\bar{s}) & =\frac{1+\lambda_{h}^{2}-2 \lambda_{h} \cos \left[2 \Psi_{x}\left(\bar{s}, \bar{s}_{h}\right)\right]}{1-\lambda_{h}^{2}} \beta_{x}(\bar{s}) \\
\beta_{y, v}(\bar{s}) & =\frac{1+\lambda_{v}^{2}-2 \lambda_{v} \cos \left[2 \Psi_{y}\left(\bar{s}, \bar{s}_{v}\right)\right]}{1-\lambda_{v}^{2}} \beta_{y}(\bar{s}) \\
\alpha_{x, h}(\bar{s}) & =\frac{1+\lambda_{h}^{2}-2 \lambda_{h} \cos \left[2 \Psi_{x}\left(\bar{s}_{,}, \bar{s}_{h}\right)\right]}{1-\lambda_{h}^{2}} \alpha_{x}(\bar{s})-\frac{2 \lambda_{h} \sin \left[2 \Psi_{x}\left(\bar{s}, \bar{s}_{h}\right)\right]}{1-\lambda_{h}^{2}} \\
\alpha_{y, v}(\bar{s}) & =\frac{1+\lambda_{v}^{2}-2 \lambda_{v} \cos \left[2 \Psi_{y}\left(\bar{s}, \bar{s}_{v}\right)\right]}{1-\lambda_{v}^{2}} \alpha_{y}(\bar{s})-\frac{2 \lambda_{v} \sin \left[2 \Psi_{y}\left(\bar{s}, \bar{s}_{v}\right)\right]}{1-\lambda_{v}^{2}}
\end{aligned}
$$

and

$$
\begin{aligned}
\tan \left[\Psi_{x, h}\left(\bar{s}, \bar{s}_{h}\right)\right] & =\frac{1+\lambda_{h}}{1-\lambda_{h}} \tan \left[\Psi_{x}\left(\bar{s}, \bar{s}_{h}\right)\right] \\
\tan \left[\Psi_{y, v}\left(\bar{s}, \bar{s}_{v}\right)\right] & =\frac{1+\lambda_{v}}{1-\lambda_{v}} \tan \left[\Psi_{y}\left(\bar{s}, \bar{s}_{v}\right)\right],
\end{aligned}
$$

where $\Psi_{x}\left(\bar{s}_{2}, \bar{s}_{1}\right), \Psi_{y}\left(\bar{s}_{2}, \bar{s}_{1}\right), \Psi_{x, h}\left(\bar{s}_{2}, \bar{s}_{1}\right)$, and $\Psi_{y, v}\left(\bar{s}_{2}, \bar{s}_{1}\right)$ are shorthand notations of

$$
\begin{aligned}
\Psi_{x}\left(\bar{s}_{2}, \bar{s}_{1}\right) & =\psi_{x}\left(\bar{s}_{2}, \bar{s}_{1}\right)-\pi \nu_{x} \operatorname{sgn}\left(\bar{s}_{2}-\bar{s}_{1}\right) \\
\Psi_{y}\left(\bar{s}_{2}, \bar{s}_{1}\right) & =\psi_{y}\left(\bar{s}_{2}, \bar{s}_{1}\right)-\pi \nu_{y} \operatorname{sgn}\left(\bar{s}_{2}-\bar{s}_{1}\right) \\
\Psi_{x, h}\left(\bar{s}_{2}, \bar{s}_{1}\right) & =\psi_{x, h}\left(\bar{s}_{2}, \bar{s}_{1}\right)-\pi \nu_{x, h} \operatorname{sgn}\left(\bar{s}_{2}-\bar{s}_{1}\right) \\
\Psi_{y, v}\left(\bar{s}_{2}, \bar{s}_{1}\right) & =\psi_{y, v}\left(\bar{s}_{2}, \bar{s}_{1}\right)-\pi \nu_{y, v} \operatorname{sgn}\left(\bar{s}_{2}-\bar{s}_{1}\right) .
\end{aligned}
$$

The arguments of the phases $\Psi_{x}\left(\bar{s}_{2}, \bar{s}_{1}\right), \Psi_{y}\left(\bar{s}_{2}, \bar{s}_{1}\right), \Psi_{x, h}\left(\bar{s}_{2}, \bar{s}_{1}\right)$, and $\Psi_{y, v}\left(\bar{s}_{2}, \bar{s}_{1}\right), \bar{s}_{1}$ and $\bar{s}_{2}$, must be in the range between 0 and $C$ unlike those of $\psi_{x}\left(s_{2}, s_{1}\right), \psi_{y}\left(s_{2}, s_{1}\right), \psi_{x, h}\left(s_{2}, s_{1}\right)$, and $\psi_{y, v}\left(s_{2}, s_{1}\right)$ can be any real number. As the case of the free motion, $\nu_{x, h}$ and $\psi_{x, h}\left(s_{2}, s_{1}\right)$ satisfy $\nu_{x, h}=\psi_{x, h}(s+C, s) / 2 \pi$ and $\nu_{y, v}$ and $\psi_{y, v}\left(s_{2}, s_{1}\right)$ satisfy $\nu_{y, v}=\psi_{y, v}(s+C, s) / 2 \pi$. Equations (28) and (32) and Equations (29) and (33) can be combined into the following complex forms:

$$
\begin{aligned}
\sqrt{\beta_{x, h}(\bar{s})} e^{-i \Psi_{x, h}\left(\bar{s}, \bar{s}_{h}\right)} & =\sqrt{\frac{\beta_{x}(\bar{s})}{1-\lambda_{h}^{2}}}\left[e^{-i \Psi_{x}\left(\bar{s}, \bar{s}_{h}\right)}-\lambda_{h} e^{i \Psi_{x}\left(\bar{s}, \bar{s}_{h}\right)}\right] \\
\sqrt{\beta_{y, v}(\bar{s})} e^{-i \Psi_{y, v}\left(\bar{s}, \bar{s}_{v}\right)} & =\sqrt{\frac{\beta_{y}(\bar{s})}{1-\lambda_{v}^{2}}}\left[e^{-i \Psi_{y}\left(\bar{s}, \bar{s}_{v}\right)}-\lambda_{v} e^{i \Psi_{y}\left(\bar{s}, \bar{s}_{v}\right)}\right] .
\end{aligned}
$$

These equations can be generalized into

$$
\begin{aligned}
\sqrt{\beta_{x, h}\left(\bar{s}_{0}\right)} e^{i \Psi_{x, h}\left(\bar{s}, \bar{s}_{0}\right)} & =\left\{e^{i\left[\psi_{x, h}\left(\bar{s}, \bar{s}_{h}\right)-\psi_{x}\left(\bar{s}, \bar{s}_{h}\right)\right]}-2 i \sin \left(\pi \delta_{h}\right) e^{i\left[\Psi_{x, h}\left(\bar{s}, \bar{s}_{h}\right)-\Psi_{x}\left(\bar{s}, \bar{s}_{h}\right)\right]} \Theta\left(\bar{s}_{0} ; \bar{s}, \bar{s}_{h}\right)\right\} \sqrt{\frac{\beta_{x}\left(\bar{s}_{0}\right)}{1-\lambda_{h}^{2}}} e^{i \Psi_{x}\left(\bar{s}, \bar{s}_{0}\right)} \\
& -\left\{\lambda_{h} e^{i\left[\psi_{x, h}\left(\bar{s}, \bar{s}_{h}\right)+\psi_{x}\left(\bar{s}, \bar{s}_{h}\right)\right]}-2 i \sin \left(\pi \delta_{h}\right) e^{i\left[\Psi_{x, h}\left(\bar{s}, \bar{s}_{h}\right)+\Psi_{x}\left(\bar{s}, \bar{s}_{h}\right)\right]} \Theta\left(\bar{s}_{0} ; \bar{s}_{,}, \bar{s}_{h}\right)\right\} \sqrt{\frac{\beta_{x}\left(\bar{s}_{0}\right)}{1-\lambda_{h}^{2}}} e^{-i \Psi_{x}\left(\bar{s}, \bar{s}_{0}\right)}
\end{aligned}
$$


and

$$
\begin{aligned}
\sqrt{\beta_{y, v}\left(\bar{s}_{0}\right)} e^{i \Psi_{y, v}\left(\bar{s}, \bar{s}_{0}\right)} & =\left\{e^{i\left[\psi_{y, v}\left(\bar{s}, \bar{s}_{v}\right)-\psi_{y}\left(\bar{s}, \bar{s}_{v}\right)\right]}-2 i \sin \left(\pi \delta_{v}\right) e^{i\left[\Psi_{y, v}\left(\bar{s}, \bar{s}_{v}\right)-\Psi_{y}\left(\bar{s}, \bar{s}_{v}\right)\right]} \Theta\left(\overline{s_{0}} ; \bar{s}, \bar{s}_{v}\right)\right\} \sqrt{\frac{\beta_{y}\left(\bar{s}_{0}\right)}{1-\lambda_{v}^{2}}} e^{i \Psi_{y}\left(\bar{s}, \bar{s}_{0}\right)} \\
& -\left\{\lambda_{v} e^{i\left[\psi_{y, v}\left(\bar{s}, \bar{s}_{v}\right)+\psi_{y}\left(\bar{s}, \bar{s}_{v}\right)\right]}-2 i \sin \left(\pi \delta_{v}\right) e^{i\left[\Psi_{y, v}\left(\bar{s}, \bar{s}_{v}\right)+\Psi_{y}\left(\bar{s}, \bar{s}_{v}\right)\right]} \Theta\left(\overline{s_{0}} ; \bar{s}, \bar{s}_{v}\right)\right\} \sqrt{\frac{\beta_{y}\left(\bar{s}_{0}\right)}{1-\lambda_{v}^{2}}} e^{-i \Psi_{y}\left(\bar{s}, \bar{s}_{0}\right)}
\end{aligned}
$$

where $\bar{s}_{0}\left(0 \leq \bar{s}_{0}<C\right)$ is an arbitrary location and $\Theta\left(s ; s_{2}, s_{1}\right)$ is a rectangular function defined as

$$
\Theta\left(s ; s_{2}, s_{1}\right)=\frac{1}{2}\left[\operatorname{sgn}\left(s_{2}-s\right)+\operatorname{sgn}\left(s-s_{1}\right)\right]=\left\{\begin{array}{ll}
1 & \left(s_{2}>s>s_{1}\right) \\
-1 & \left(s_{1}>s>s_{2}\right) \\
0 & (\text { other cases })
\end{array} .\right.
$$

These two equations are used in Section 3. Equations (20), (21), (22), and (23) indicate that the optical parameters measured directly from an observation of the driven motion are $\beta_{x, h}(\bar{s}), \beta_{y, v}(\bar{s}), \alpha_{x, h}(\bar{s}), \alpha_{y, v}(\bar{s}), \psi_{x, h}\left(\bar{s}_{2}, s_{1}\right)$, and $\psi_{y, v}\left(\bar{s}_{2}, s_{1}\right)$. To determine the optical parameters of the free motion, we need to use Equations (28), (29), (30), (31), (32), and (33) with these measured $\beta_{x, h}(\bar{s}), \beta_{y, v}(\bar{s}), \alpha_{x, h}(\bar{s}), \alpha_{y, v}(\bar{s}), \psi_{x, h}\left(\bar{s}_{2}, s_{1}\right)$, and $\psi_{y, v}\left(\bar{s}_{2}, s_{1}\right)$. These apparent modulations of the optical parameters are due to the sum resonances (assuming the difference resonance is dominant), whose modes rotate in the opposite direction compared to the modes of the difference resonances. We may see that, when rewriting Equations (16) and (17) into (20) and (21), the terms of the sum resonances are absorbed into the new optical parameters. The sum resonances become negligible compared to the difference resonances in the limit $\delta_{h}, \delta_{v} \rightarrow 0$. Then, from Equations (28), (29), (30), (31), (32), and (33), $\beta_{x, h}(\bar{s}), \beta_{y, v}(\bar{s}), \alpha_{x, h}(\bar{s}), \alpha_{y, v}(\bar{s}), \psi_{x, h}\left(\bar{s}_{2}, \bar{s}_{1}\right)$ and $\psi_{y, v}\left(\bar{s}_{2}, \bar{s}_{1}\right)$ converge to $\beta_{x}(\bar{s}), \beta_{y}(\bar{s}), \alpha_{x}(\bar{s}), \alpha_{y}(\bar{s}), \psi_{x}\left(\bar{s}_{2}, \bar{s}_{1}\right)$, and $\psi_{y}\left(\bar{s}_{2}, \bar{s}_{1}\right)$. However, it may be hard to achieve this limiting case in a real ring, particularly in a hadron ring. It has been shown that these modulations of the optical parameters are equivalent to the case when thin quadrupole fields, which change the tunes by $\delta_{h}$ and $\delta_{v}$, are inserted at the locations of the AC dipoles [5].

Equations (20), (21), (22), and (23) also indicate that complex representations of the phase spaces suited to the driven motion are not $\tilde{x}=x+i\left[\alpha_{x}(\bar{s}) x+\beta_{x}(\bar{s}) x^{\prime}\right]$ and $\tilde{y}=y+i\left[\alpha_{y}(\bar{s}) y+\beta_{y}(\bar{s}) y^{\prime}\right]$ but $\tilde{x}_{h}=x+i\left[\alpha_{x, h}(\bar{s}) x+\beta_{x, h}(\bar{s}) x^{\prime}\right]$ and $\tilde{y}_{v}=y+i\left[\alpha_{y, v}(\bar{s}) y+\beta_{y, v}(\bar{s}) y^{\prime}\right]$. In these representations, phase space positions of the driven motion are given by

$$
\begin{aligned}
& \tilde{x}_{h}(n ; \bar{s})=A_{x, h} \sqrt{\beta_{x, h}(\bar{s})} e^{-2 \pi i \nu_{x, h} n-i \psi_{x, h}\left(\bar{s}_{,}, \bar{s}_{h}\right)-i \phi_{h}} \\
& \tilde{y}_{v}(n ; \bar{s})=A_{y, v} \sqrt{\beta_{y, v}(\bar{s})} e^{-2 \pi i \nu_{y, v} n-i \psi_{y, v}\left(\bar{s}, \bar{s}_{v}\right)-i \phi_{v}} .
\end{aligned}
$$

The relations of the complex representations between $\tilde{x}$ and $\tilde{x}_{h}$ and between $\tilde{y}$ and $\tilde{y}_{v}$ are given by

$$
\begin{gathered}
\tilde{x}_{h}=\sqrt{\frac{1}{1-\lambda_{h}^{2}} \frac{\beta_{x, h}(\bar{s})}{\beta_{x}(\bar{s})}}\left\{e^{-i\left[\Psi_{x, h}\left(\bar{s}_{,} \bar{s}_{h}\right)-\Psi_{x}\left(\bar{s}, \bar{s}_{h}\right)\right]} \tilde{x}+\lambda_{h} e^{-i\left[\Psi_{x, h}\left(\bar{s}, \bar{s}_{h}\right)+\Psi_{x}\left(\bar{s}, \bar{s}_{h}\right)\right]} \tilde{x}^{*}\right\} \\
\tilde{y}_{v}=\sqrt{\frac{1}{1-\lambda_{v}^{2}} \frac{\beta_{y, v}(\bar{s})}{\beta_{y}(\bar{s})}}\left\{e^{-i\left[\Psi_{y, v}\left(\bar{s}, \bar{s}_{v}\right)-\Psi_{y}\left(\bar{s}, \bar{s}_{v}\right)\right]} \tilde{y}+\lambda_{v} e^{-i\left[\Psi_{y, v}\left(\bar{s}, \bar{s}_{v}\right)+\Psi_{y}\left(\bar{s}, \bar{s}_{v}\right)\right]} \tilde{y}^{*}\right\} .
\end{gathered}
$$

For the driven motion, the complex phase space positions constructed with two adjacent BPMs are also $\tilde{x}_{h}$ and $\tilde{y}_{v}$ instead of $\tilde{x}$ and $\tilde{y}$. Similar to Equations (12) and (13), the following equations hold:

$$
\begin{aligned}
& \tilde{x}_{h}\left(n ; \bar{s}_{1}\right)=\frac{1}{i \sin \left[\psi_{x, h}\left(\bar{s}_{2}, \bar{s}_{1}\right)\right]}\left[e^{i \psi_{x, h}\left(\bar{s}_{2}, \bar{s}_{1}\right)} x\left(n ; \bar{s}_{1}\right)-\sqrt{\left.\frac{\beta_{x, h}\left(\bar{s}_{1}\right)}{\beta_{x, h}\left(\bar{s}_{2}\right)} x\left(n ; \bar{s}_{2}\right)\right]}\right. \\
& \tilde{y}_{v}\left(n ; \bar{s}_{1}\right)=\frac{1}{i \sin \left[\psi_{y, v}\left(\bar{s}_{2}, \bar{s}_{1}\right)\right]}\left[e^{i \psi_{y, v}\left(\bar{s}_{2}, \bar{s}_{1}\right)} y\left(n ; \bar{s}_{1}\right)-\sqrt{\left.\frac{\beta_{y, v}\left(\bar{s}_{1}\right)}{\beta_{y, v}\left(\bar{s}_{2}\right)} y\left(n ; \bar{s}_{2}\right)\right] .}\right.
\end{aligned}
$$

\subsection{Coupled Free Betatron Motion}

The first order effects of skew quadrupole fields on the betatron motion can be calculated in various ways. As we see in the following, the skew quadrupole fields behave like AC dipoles on the first order and one way to calculate their effects is to use this analogy. In this section, we present a solution to calculate CRDT based on this analogy. A similar solution can be applied when we calculate the effect of the skew quadrupole fields on the driven motion in the next section. 
When a ring has $N$ thin skew quadrupole fields at locations $\bar{s}_{j}(j=1 \cdots N)$ and their effective strengths are $\kappa_{j}$, where

$$
\kappa_{j}=\left.\frac{1}{(B \rho)} \frac{\partial B_{x}\left(x, y, \bar{s}_{j}\right)}{\partial x}\right|_{x=y=0} \times(\text { length of the field }),
$$

from Equations (1) and (2), the equations of motion are

$$
\begin{aligned}
& \frac{d^{2} x}{d s^{2}}+K_{x}(\bar{s}) x=\left[\sum_{j=1}^{N} \sum_{m=-\infty}^{\infty} \kappa_{j} \delta\left(s-\bar{s}_{j}-m C\right)\right] y \\
& \frac{d^{2} y}{d s^{2}}+K_{y}(\bar{s}) y=\left[\sum_{j=1}^{N} \sum_{m=-\infty}^{\infty} \kappa_{j} \delta\left(s-\bar{s}_{j}-m C\right)\right] x .
\end{aligned}
$$

We solve these equations by expanding the solutions according to the order of the skew quadrupole fields:

$$
\begin{aligned}
& x(s)=x^{(0)}(s)+x^{(1)}(s)+\cdots \\
& y(s)=y^{(0)}(s)+y^{(1)}(s)+\cdots,
\end{aligned}
$$

where the superscripts denote the orders. The leading order solutions, $x^{(0)}(s)$ and $y^{(0)}(s)$, are obviously Equations $(4)$ and (5). The equations to determine the first order solutions, $x^{(1)}(s)$ and $y^{(1)}(s)$, are given by substituting Equations (4), (5), (52), and (53) into Equations (50) and (51):

$$
\begin{aligned}
\frac{d^{2} x^{(1)}}{d s^{2}}+K_{x}(\bar{s}) x^{(1)} & =\left[\sum_{j=1}^{N} \sum_{m=-\infty}^{\infty} \kappa_{j} \delta\left(s-\bar{s}_{j}-m C\right)\right] y^{(0)}(s) \\
& =\sum_{j=1}^{N} \sum_{m=-\infty}^{\infty} A_{y} \kappa_{j} \sqrt{\beta_{y}\left(\bar{s}_{j}\right)} \delta\left(s-\bar{s}_{j}-m C\right) \cos \left[2 \pi \nu_{y} m+\psi_{y}\left(\bar{s}_{j}\right)+\phi_{y}\right]
\end{aligned}
$$

and

$$
\begin{aligned}
\frac{d^{2} y^{(1)}}{d s^{2}}+K_{y}(\bar{s}) y^{(1)} & =\left[\sum_{j=1}^{N} \sum_{m=-\infty}^{\infty} \kappa_{j} \delta\left(s-\bar{s}_{j}-m C\right)\right] x^{(0)}(s) \\
& =\sum_{j=1}^{N} \sum_{m=-\infty}^{\infty} A_{x} \kappa_{j} \sqrt{\beta_{x}\left(\bar{s}_{j}\right)} \delta\left(s-\bar{s}_{j}-m C\right) \cos \left[2 \pi \nu_{x} m+\psi_{x}\left(\bar{s}_{j}\right)+\phi_{x}\right]
\end{aligned}
$$

Comparing these equations to the equations of motion when there are AC dipoles, Equations (14) and (15), we may see that each skew quadrupole field acts like an AC dipole. Hence, the contribution of the $j$ th skew quadrupole field to $x^{(1)}(s)$ is given by simply replacing $\theta_{h}, \bar{s}_{h}, \nu_{x, h}$, and $\phi_{h}$ with $\left[-A_{y} \kappa_{j} \sqrt{\beta_{y}\left(\bar{s}_{j}\right)}\right], \bar{s}_{j}, \nu_{y}$, and $\left[\psi_{y}\left(\bar{s}_{j}\right)+\phi_{y}\right]$ in Equation (16). Similarly, the contribution of the $j$ th skew quadrupole field to $y^{(1)}(s)$ is given by replacing $\theta_{v}, \bar{s}_{v}, \nu_{y, v}$, and $\phi_{v}$ with $\left[-A_{x} \kappa_{j} \sqrt{\beta_{x}\left(\bar{s}_{j}\right)}\right], \bar{s}_{j}, \nu_{x}$, and $\left[\psi_{x}\left(\bar{s}_{j}\right)+\phi_{x}\right]$ in Equation (17). The first order solutions, $x^{(1)}(s)$ and $y^{(1)}(s)$, are linear superpositions of such contributions from each skew quadrupole field. After some algebraic calculations, the first order solutions in the complex representation are given by

$$
\begin{aligned}
& \tilde{x}^{(1)}(n ; \bar{s})=2 i A_{y} f_{-}(\bar{s}) \sqrt{\beta_{x}(\bar{s})} e^{-2 \pi i \nu_{y} n-i \psi_{y}(\bar{s})-i \phi_{y}}+2 i A_{y} f_{+}(\bar{s}) \sqrt{\beta_{x}(\bar{s})} e^{2 \pi i \nu_{y} n+i \psi_{y}(\bar{s})+i \phi_{y}} \\
& \tilde{y}^{(1)}(n ; \bar{s})=2 i A_{x} f_{-}^{*}(\bar{s}) \sqrt{\beta_{y}(\bar{s})} e^{-2 \pi i \nu_{x} n-i \psi_{x}(\bar{s})-i \phi_{x}}+2 i A_{x} f_{+}(\bar{s}) \sqrt{\beta_{y}(\bar{s})} e^{2 \pi i \nu_{x} n+i \psi_{x}(\bar{s})+i \phi_{x}},
\end{aligned}
$$

where $f_{\mp}(\bar{s})$ is a CRDT defined as

$$
f_{\mp}(\bar{s})=\frac{1}{8 i \sin \left[\pi\left(\nu_{x} \mp \nu_{y}\right)\right]} \sum_{j=1}^{N} \kappa_{j} \sqrt{\beta_{x}\left(\bar{s}_{j}\right) \beta_{y}\left(\bar{s}_{j}\right)} e^{-i\left[\Psi_{x}\left(\bar{s}, \bar{s}_{j}\right) \mp \Psi_{y}\left(\bar{s}, \bar{s}_{j}\right)\right]} .
$$

This derivation assumes that the effective strengths of the skew quadrupole fields, $\kappa_{j}$, are adiabatic parameters. In other words, Equations (56) and (57) are steady state solutions. If the strengths of these skew quadrupole fields are suddenly 
turned from zero to finite values or when a beam is injected to the ring, additional transient modes are also excited. Similar to the parameters $\lambda_{h}$ and $\lambda_{v}$ for the driven motion in Equations (26) and (27), we define a parameter for the ratio of magnitudes of the difference and sum resonances:

$$
\lambda_{c}=\frac{\sin \left[\pi\left(\nu_{x}-\nu_{y}\right)\right]}{\sin \left[\pi\left(\nu_{x}+\nu_{y}\right)\right]} .
$$

In the following, we assume that fractional parts of the horizontal and vertical tunes are close to each other and $\left|\lambda_{c}\right| \ll 1$.

The CRDTs at the location of one dual-plane BPM, $\bar{s}$, are determined from Fourier components of the turn-byturn motion observed by this BPM. The Fourier components corresponding to the leading order modes, $\mathscr{X}\left(-\nu_{x} ; \bar{s}\right)$ and $\mathscr{Y}\left(-\nu_{y} ; \bar{s}\right)$, are already given in Equations (10) and (11). From Equations (56) and (57), the Fourier components corresponding to the first order modes are

$$
\begin{aligned}
\mathscr{X}\left(-\nu_{y} ; \bar{s}\right) & =2 i A_{y} f_{-}(\bar{s}) \sqrt{\beta_{x}(\bar{s})} e^{-i \psi_{y}(\bar{s})-i \phi_{y}} \\
\mathscr{X}\left(\nu_{y} ; \bar{s}\right) & =2 i A_{y} f_{+}(\bar{s}) \sqrt{\beta_{x}(\bar{s})} e^{i \psi_{y}(\bar{s})+i \phi_{y}} \\
\mathscr{Y}\left(-\nu_{x} ; \bar{s}\right) & =2 i A_{x} f_{-}^{*}(\bar{s}) \sqrt{\beta_{y}(\bar{s})} e^{-i \psi_{x}(\bar{s})-i \phi_{x}} \\
\mathscr{Y}\left(\nu_{x} ; \bar{s}\right) & =2 i A_{x} f_{+}(\bar{s}) \sqrt{\beta_{y}(\bar{s})} e^{i \psi_{x}(\bar{s})+i \phi_{x}} .
\end{aligned}
$$

From Equations $(10),(11),(60),(61),(62)$, and $(63), f_{\mp}(\bar{s})$ are given by

$$
\begin{aligned}
& f_{-}(\bar{s})=\frac{1}{-2 i} \sqrt{\frac{\beta_{x}(\bar{s})}{\beta_{y}(\bar{s})}} \frac{\mathscr{Y}^{*}\left(-\nu_{x} ; \bar{s}\right)}{\mathscr{X}^{*}\left(-\nu_{x} ; \bar{s}\right)}=\frac{1}{2 i} \sqrt{\frac{\beta_{y}(\bar{s})}{\beta_{x}(\bar{s})}} \frac{\mathscr{X}\left(-\nu_{y} ; \bar{s}\right)}{\mathscr{Y}\left(-\nu_{y} ; \bar{s}\right)} \\
& f_{+}(\bar{s})=\frac{1}{2 i} \sqrt{\frac{\beta_{x}(\bar{s})}{\beta_{y}(\bar{s})}} \frac{\mathscr{Y}\left(\nu_{x} ; \bar{s}\right)}{\mathscr{X}^{*}\left(-\nu_{x} ; \bar{s}\right)}=\frac{1}{2 i} \sqrt{\frac{\beta_{y}(\bar{s})}{\beta_{x}(\bar{s})}} \frac{\mathscr{X}\left(\nu_{y} ; \bar{s}\right)}{\mathscr{Y}^{*}\left(-\nu_{y} ; \bar{s}\right)} .
\end{aligned}
$$

If the horizontal and vertical pick-ups of the considered dual-plane BPM have a calibration error, it affects inversely to the first and second expressions of $f_{\mp}(\bar{s})$. Hence, to cancel the potential calibration errors, we usually take the square average of the first and second expressions when calculating amplitude of $f_{\mp}(\bar{s})[10]$ :

$$
\begin{aligned}
\left|f_{-}(\bar{s})\right| & =\frac{1}{2} \sqrt{-\frac{\mathscr{Y}\left(-\nu_{x} ; \bar{s}\right) \mathscr{X}\left(-\nu_{y} ; \bar{s}\right)}{\mathscr{X}\left(-\nu_{x} ; \bar{s}\right) \mathscr{Y}\left(-\nu_{y} ; \bar{s}\right)}} \\
\left|f_{+}(\bar{s})\right| & =\frac{1}{2} \sqrt{\frac{\mathscr{Y} *\left(\nu_{x} ; \bar{s}\right) \mathscr{X}\left(\nu_{y} ; \bar{s}\right)}{\mathscr{X}\left(-\nu_{x} ; \bar{s}\right) \mathscr{Y} *\left(-\nu_{y} ; \bar{s}\right)}} .
\end{aligned}
$$

When $f_{\mp}(\bar{s})$ at two BPM locations $\bar{s}_{1}$ and $\bar{s}_{2}\left(\bar{s}_{1}<\bar{s}_{2}\right)$ are known, we can get an information of the skew quadrupole fields located between these two BPMs from the following equation [11]:

$$
e^{i\left[\psi_{x}\left(\bar{s}_{2}\right) \mp \psi_{y}\left(\bar{s}_{2}\right)\right]} f_{\mp}\left(\bar{s}_{2}\right)-e^{i\left[\psi_{x}\left(\bar{s}_{1}\right) \mp \psi_{y}\left(\bar{s}_{1}\right)\right]} f_{\mp}\left(\bar{s}_{1}\right)=\sum_{\bar{s}_{1}<\bar{s}_{j}<\bar{s}_{2}} \frac{1}{4} \kappa_{j} \sqrt{\beta_{x}\left(\bar{s}_{j}\right) \beta_{y}\left(\bar{s}_{j}\right)} e^{i\left[\psi_{x}\left(\bar{s}_{j}\right) \mp \psi_{y}\left(\bar{s}_{j}\right)\right]} .
$$

\section{Coupled Driven Betatron Motion}

In this section, we derive an expression of the coupled driven betatron motion, excited with two AC dipoles under the influence of skew quadrupole fields. Similar to the case of the uncoupled driven motion in Section 2.2, which is parametrized with the modulated Courant-Snyder parameters and phase advance, the coupled driven motion is parametrized with modulated CRDTs. The procedure to calculate the first order effects of the skew quadrupole fields on the driven motion is almost identical to that on the free motion in Section 2.3. From Equations (14), (15), (50), and (51), equations of motion when there are horizontal and vertical AC dipoles and skew quadrupole fields are given by

$$
\begin{aligned}
& \frac{d^{2} x}{d s^{2}}+K_{x}(\bar{s}) x=\left[\sum_{j=1}^{N} \sum_{m=-\infty}^{\infty} \kappa_{j} \delta\left(s-\bar{s}_{j}-m C\right)\right] y-\sum_{m=-\infty}^{\infty} \theta_{h} \delta\left(s-\bar{s}_{h}-m C\right) \cos \left(2 \pi \nu_{x}, h m+\phi_{h}\right) \\
& \frac{d^{2} y}{d s^{2}}+K_{y}(\bar{s}) y=\left[\sum_{j=1}^{N} \sum_{m=-\infty}^{\infty} \kappa_{j} \delta\left(s-\bar{s}_{j}-m C\right)\right] x-\sum_{m=-\infty}^{\infty} \theta_{v} \delta\left(s-\bar{s}_{v}-m C\right) \cos \left(2 \pi \nu_{y, v} m+\phi_{v}\right) .
\end{aligned}
$$


As we did in Section 2.3, we solve these equations by expanding the solutions according to the order of the skew quadrupole fields. The leading order solutions, $x^{(0)}(s)$ and $y^{(0)}(s)$, are already given in Equations $(20)$ and $(21)$. Then, the equations to determine the first order solutions, $x^{(1)}(s)$ and $y^{(1)}(s)$, are given by

$$
\begin{aligned}
\frac{d^{2} x^{(1)}}{d s^{2}}+K_{x}(\bar{s}) x^{(1)} & =\left[\sum_{j=1}^{N} \sum_{m=-\infty}^{\infty} \kappa_{j} \delta\left(s-\bar{s}_{j}-m C\right)\right] y^{(0)}(s) \\
& =\sum_{j=1}^{N} \sum_{m=-\infty}^{\infty} A_{y, v} \kappa_{j} \sqrt{\beta_{y, v}\left(\bar{s}_{j}\right)} \delta\left(s-\bar{s}_{j}-m C\right) \cos \left[2 \pi \nu_{y, v} m+\psi_{y, v}\left(\bar{s}_{j}, \bar{s}_{v}\right)+\phi_{v}\right]
\end{aligned}
$$

and

$$
\begin{aligned}
\frac{d^{2} y^{(1)}}{d s^{2}}+K_{y}(\bar{s}) y^{(1)} & =\left[\sum_{j=1}^{N} \sum_{m=-\infty}^{\infty} \kappa_{j} \delta\left(s-\bar{s}_{j}-m C\right)\right] x^{(0)}(s) \\
& =\sum_{j=1}^{N} \sum_{m=-\infty}^{\infty} A_{x, h} \kappa_{j} \sqrt{\beta_{x, h}\left(\bar{s}_{j}\right)} \delta\left(s-\bar{s}_{j}-m C\right) \cos \left[2 \pi \nu_{x, h} m+\psi_{x, h}\left(\bar{s}_{j}, \bar{s}_{h}\right)+\phi_{h}\right]
\end{aligned}
$$

These equations can be solved in the same way as we solved Equations (54) and (55) by replacing the corresponding terms in Equations (16) and (17). After some algebraic calculations, the first order solutions in the complex representations are given by

$$
\begin{aligned}
& \tilde{x}^{(1)}(n ; \bar{s})=2 i A_{y, v} f_{-, v}(\bar{s}) \sqrt{\beta_{x}(\bar{s})} e^{-2 \pi i \nu_{y, v} n-i \psi_{y, v}\left(\bar{s}, \bar{s}_{v}\right)-i \phi_{v}}+2 i A_{y, v} f_{+, v}(\bar{s}) \sqrt{\beta_{x}(\bar{s})} e^{2 \pi i \nu_{y, v} n+i \psi_{y, v}\left(\bar{s}, \bar{s}_{v}\right)+i \phi_{v}} \\
& \tilde{y}^{(1)}(n ; \bar{s})=2 i A_{x, h} f_{-, h}^{*}(\bar{s}) \sqrt{\beta_{y}(\bar{s})} e^{-2 \pi i \nu_{x, h} n-i \psi_{x, h}\left(\bar{s}, \bar{s}_{h}\right)-i \phi_{h}}+2 i A_{x, h} f_{+, h}(\bar{s}) \sqrt{\beta_{y}(\bar{s})} e^{2 \pi i \nu_{x, h} n+i \psi_{x, h}\left(\bar{s}, \bar{s}_{h}\right)+i \phi_{h}},
\end{aligned}
$$

where $f_{\mp, h}(\bar{s})$ and $f_{\mp, v}(\bar{s})$ are CRDTs for the driven motion define as

$$
\begin{aligned}
& f_{\mp, h}(\bar{s})=\frac{1}{8 i \sin \left[\pi\left(\nu_{x, h} \mp \nu_{y}\right)\right]} \sum_{j=1}^{N} \kappa_{j} \sqrt{\beta_{x, h}\left(\bar{s}_{j}\right) \beta_{y}\left(\bar{s}_{j}\right)} e^{-i\left[\Psi_{x, h}\left(\bar{s}, \bar{s}_{j}\right) \mp \Psi_{y}\left(\bar{s}, \bar{s}_{j}\right)\right]} \\
& f_{\mp, v}(\bar{s})=\frac{1}{8 i \sin \left[\pi\left(\nu_{x} \mp \nu_{y, v}\right)\right]} \sum_{j=1}^{N} \kappa_{j} \sqrt{\beta_{x}\left(\bar{s}_{j}\right) \beta_{y, v}\left(\bar{s}_{j}\right)} e^{-i\left[\Psi_{x}\left(\bar{s}, \bar{s}_{j}\right) \mp \Psi_{y, v}\left(\bar{s}, \bar{s}_{j}\right)\right]} .
\end{aligned}
$$

Compared to the CRDTs for the free motion, $f_{\mp}(\bar{s})$, in Equation $(58)$, the optical parameters in the horizontal plane are replaced by those for the driven motion in $f_{\mp, h}(\bar{s})$ and the optical parameters in the vertical plane are replaced by those for the driven motion in $f_{\mp, v}(\bar{s})$. In this way, the coupled driven motion is parametrized with a new set of CRDTs. If we use Equations (40) and (41) and rewrite $\left[\sqrt{\beta_{x, h}\left(\bar{s}_{j}\right)} e^{-i \Psi_{x, h}\left(\bar{s}, \bar{s}_{j}\right)}\right]$ in Equation $(75)$ and $\left[\sqrt{\beta_{y, v}\left(\bar{s}_{j}\right)} e^{ \pm i \Psi_{y, v}\left(\bar{s}_{,} \bar{s}_{j}\right)}\right]$ in Equation $(76), f_{\mp, h}(\bar{s})$ and $f_{\mp, v}(\bar{s})$ can be expressed with $f_{\mp}(\bar{s})$ :

$$
\begin{aligned}
f_{\mp, h}(\bar{s})= & \frac{1}{\sqrt{1-\lambda_{h}^{2}}} \frac{\sin \left[\pi\left(\nu_{x} \mp \nu_{y}\right)\right]}{\sin \left[\pi\left(\nu_{x, h} \mp \nu_{y}\right)\right]}\left\{e^{-i\left[\psi_{x, h}\left(\bar{s}, \bar{s}_{h}\right)-\psi_{x}\left(\bar{s}, \bar{s}_{h}\right)\right]} f_{\mp}(\bar{s})+\lambda_{h} e^{-i\left[\psi_{x, h}\left(\bar{s}, \bar{s}_{h}\right)+\psi_{x}\left(\bar{s}, \bar{s}_{h}\right)\right]}\left[\lambda_{c}^{\mp 1} f_{ \pm}^{*}(\bar{s})\right]\right. \\
& \left.+2 i \sin \left(\pi \delta_{h}\right) e^{-i\left[\Psi_{x, h}\left(\bar{s}_{,}, \bar{s}_{h}\right)-\Psi_{x}\left(\bar{s}, \bar{s}_{h}\right)\right]} f_{\mp}\left(\bar{s} ; \bar{s}_{,} \bar{s}_{h}\right)+2 i \sin \left(\pi \delta_{h}\right) e^{-i\left[\Psi_{x, h}\left(\bar{s}, \bar{s}_{h}\right)+\Psi_{x}\left(\bar{s}_{,}, \bar{s}_{h}\right)\right]}\left[\lambda_{c}^{\mp 1} f_{ \pm}^{*}\left(\bar{s} ; \bar{s}, \bar{s}_{h}\right)\right]\right\}
\end{aligned}
$$

and

$$
\begin{aligned}
f_{\mp, v}(\bar{s})= & \frac{1}{\sqrt{1-\lambda_{v}^{2}}} \frac{\sin \left[\pi\left(\nu_{x} \mp \nu_{y}\right)\right]}{\sin \left[\pi\left(\nu_{x} \mp \nu_{y, v}\right)\right]}\left\{e^{ \pm i\left[\psi_{y, v}\left(\bar{s}, \bar{s}_{v}\right)-\psi_{y}\left(\bar{s}, \bar{s}_{v}\right)\right]} f_{\mp}(\bar{s})-\lambda_{v} e^{ \pm i\left[\psi_{y, v}\left(\bar{s}, \bar{s}_{v}\right)+\psi_{y}\left(\bar{s}, \bar{s}_{v}\right)\right]}\left[\lambda_{c}^{\mp 1} f_{ \pm}(\bar{s})\right]\right. \\
& \left.\mp 2 i \sin \left(\pi \delta_{v}\right) e^{ \pm i\left[\Psi_{y, v}\left(\bar{s}, \bar{s}_{v}\right)-\Psi_{y}\left(\bar{s}_{,}, \bar{s}_{v}\right)\right]} f_{\mp}\left(\bar{s} ; \bar{s}, \bar{s}_{v}\right) \pm 2 i \sin \left(\pi \delta_{v}\right) e^{ \pm i\left[\Psi_{y, v}\left(\bar{s}, \bar{s}_{v}\right)+\Psi_{y}\left(\bar{s}_{,}, \bar{s}_{v}\right)\right]}\left[\lambda_{c}^{\mp 1} f_{ \pm}\left(\bar{s} ; \bar{s}, \bar{s}_{v}\right)\right]\right\}
\end{aligned}
$$

where $f_{\mp}\left(\bar{s} ; \bar{s}, \bar{s}_{h}\right)$ and $f_{\mp}\left(\bar{s} ; \bar{s}, \bar{s}_{v}\right)$ describe contributions to $f_{\mp}(\bar{s})$ from the skew quadrupole fields located between $\bar{s}_{h}$ and $\bar{s}$ and between $\bar{s}_{v}$ and $\bar{s}$ :

$$
f_{\mp}\left(\bar{s} ; \bar{s}, \bar{s}_{\mathrm{ac}}\right)=\frac{1}{8 i \sin \left[\pi\left(\nu_{x} \mp \nu_{y}\right)\right]} \sum_{j=1}^{N} \Theta\left(\bar{s}_{j} ; \bar{s}, \bar{s}_{\mathrm{ac}}\right) \kappa_{j} \sqrt{\beta_{x}\left(\bar{s}_{j}\right) \beta_{y}\left(\bar{s}_{j}\right)} e^{-i\left[\Psi_{x}\left(\bar{s}, \bar{s}_{j}\right) \mp \Psi_{y}\left(\bar{s}, \bar{s}_{j}\right)\right]} \quad\left(\bar{s}_{\mathrm{ac}}=\bar{s}_{h} \text { or } \bar{s}_{v}\right) .
$$


If we use Equation $(68), f_{\mp}\left(\bar{s} ; \bar{s}, \bar{s}_{\text {ac }}\right)$ is given with $f_{\mp}(\bar{s})$ and $f_{\mp}\left(\bar{s}_{\text {ac }}\right)$ :

$$
f_{\mp}\left(\bar{s} ; \bar{s}, \bar{s}_{\mathrm{ac}}\right)=\frac{e^{\pi i\left(\nu_{x} \mp \nu_{y}\right) \operatorname{sgn}\left(\bar{s}-\bar{s}_{\mathrm{ac}}\right)}}{2 i \sin \left[\pi\left(\nu_{x} \mp \nu_{y}\right)\right]}\left\{f_{\mp}(\bar{s})-e^{-i\left[\psi_{x}\left(\bar{s}, \bar{s}_{\mathrm{ac}}\right) \mp \psi_{y}\left(\bar{s}, \bar{s}_{\mathrm{ac}}\right)\right]} f_{\mp}\left(\bar{s}_{\mathrm{ac}}\right)\right\} \quad\left(\bar{s}_{\mathrm{ac}}=\bar{s}_{h} \text { or } \bar{s}_{v}\right) .
$$

As we discuss in Section $4, f_{\mp, h}(\bar{s})$ and $f_{\mp, v}(\bar{s})$ can be determined from an observation of the driven motion. Hence, if the horizontal and vertical AC dipoles are at the same location in the ring, suppose $\bar{s}_{h}=\bar{s}_{v}=\bar{s}_{\text {ac }}$, Equations $(77)$ and (78) provide four equations for four known parameters, $f_{\mp, h}(\bar{s})$ and $f_{\mp, v}(\bar{s})$, and four unknown parameters, $f_{\mp}(\bar{s})$ and $f_{\mp}\left(\bar{s} ; \bar{s}, \bar{s}_{\text {ac }}\right.$ ) (or equivalently $f_{\mp}(\bar{s})$ and $f_{\mp}\left(\bar{s}_{\text {ac }}\right)$ ), and it looks that we can algebraically solve these equations for $f_{\mp}(\bar{s})$. However, this is not the case since these equations are not linearly independent. This indicates that $f_{\mp}(\bar{s})$ at one BPM location $\bar{s}$ cannot be determined only with $f_{\mp, h}(\bar{s})$ and $f_{\mp, v}(\bar{s})$ at the same location. As shown in Section 4 , we also need $f_{\mp, h}\left(\bar{s}_{h}\right)$ and $f_{\mp, v}\left(\bar{s}_{v}\right)$ to determine $f_{\mp}(\bar{s})$. Similar to Equation $(68)$, the following equations hold for $f_{\mp, h}(\bar{s})$ and $f_{\mp, v}(\bar{s})$ at two BPM locations $\bar{s}_{1}$ and $\bar{s}_{2}$ :

$$
\begin{aligned}
e^{i\left[\psi_{x, h}\left(\bar{s}_{2}\right) \mp \psi_{y}\left(\bar{s}_{2}\right)\right]} f_{\mp, h}\left(\bar{s}_{2}\right)-e^{i\left[\psi_{x, h}\left(\bar{s}_{1}\right) \mp \psi_{y}\left(\bar{s}_{1}\right)\right]} f_{\mp, h}\left(\bar{s}_{1}\right) & =\sum_{\bar{s}_{1}<\bar{s}_{j}<\bar{s}_{2}} \frac{1}{4} \kappa_{j} \sqrt{\beta_{x, h}\left(\bar{s}_{j}\right) \beta_{y}\left(\bar{s}_{j}\right)} e^{i\left[\psi_{x, h}\left(\bar{s}_{j}\right) \mp \psi_{y}\left(\bar{s}_{j}\right)\right]} \\
e^{i\left[\psi_{x}\left(\bar{s}_{2}\right) \mp \psi_{y, v}\left(\bar{s}_{2}\right)\right]} f_{\mp, v}\left(\bar{s}_{2}\right)-e^{i\left[\psi_{x}\left(\bar{s}_{1}\right) \mp \psi_{y, v}\left(\bar{s}_{1}\right)\right]} f_{\mp, v}\left(\bar{s}_{1}\right) & =\sum_{\bar{s}_{1}<\bar{s}_{j}<\bar{s}_{2}} \frac{1}{4} \kappa_{j} \sqrt{\beta_{x}\left(\bar{s}_{j}\right) \beta_{y, v}\left(\bar{s}_{j}\right)} e^{i\left[\psi_{x}\left(\bar{s}_{j}\right) \mp \psi_{y, v}\left(\bar{s}_{j}\right)\right]} .
\end{aligned}
$$

We note that the solutions in Equations (73) and (74) are in the complex phase space representations based on the parameters of the free motion, $\tilde{x}=x+i\left[\alpha_{x}(\bar{s}) x+\beta_{x}(\bar{s}) x^{\prime}\right]$ and $\tilde{y}=y+i\left[\alpha_{y}(\bar{s}) y+\beta_{y}(\bar{s}) y^{\prime}\right]$. Whereas, as discussed in Section 2.2, the complex phase space positions reconstructed from two adjacent BPMs for the driven motion are those based on the parameters of the driven motion, $\tilde{x}_{h}=x+i\left[\alpha_{x, h}(\bar{s}) x+\beta_{x, h}(\bar{s}) x^{\prime}\right]$ and $\tilde{y}_{v}=y+i\left[\alpha_{y, v}(\bar{s}) y+\beta_{y, v}(\bar{s}) y^{\prime}\right]$. The transformations from $\tilde{x}$ and $\tilde{y}$ to $\tilde{x}_{h}$ and $\tilde{y}_{v}$ are given in Equations (45) and (45):

$$
\begin{aligned}
& \tilde{x}_{h}^{(1)}(n ; \bar{s})=2 i A_{y, v} f_{-, v, h}(\bar{s}) \sqrt{\beta_{x, h}(\bar{s})} e^{-2 \pi i \nu_{y, v} n-i \psi_{y, v}\left(\bar{s}, \bar{s}_{v}\right)-i \phi_{v}}+2 i A_{y, v} f_{+, v, h}(\bar{s}) \sqrt{\beta_{x, h}(\bar{s})} e^{2 \pi i \nu_{y, v} n+i \psi_{y, v}\left(\bar{s}, \bar{s}_{v}\right)+i \phi_{v}} \\
& \tilde{y}_{v}^{(1)}(n ; \bar{s})=2 i A_{x, h} f_{-, h, v}^{*}(\bar{s}) \sqrt{\beta_{y, v}(\bar{s})} e^{-2 \pi i \nu_{x, h} n-i \psi_{x, h}\left(\bar{s}, \bar{s}_{h}\right)-i \phi_{h}}+2 i A_{x, h} f_{+, h, v}(\bar{s}) \sqrt{\beta_{y, v}(\bar{s})} e^{2 \pi i \nu_{x, h} n+i \psi_{x, h}\left(\bar{s}, \bar{s}_{h}\right)+i \phi_{h}}
\end{aligned}
$$

where $f_{\mp, h, v}(\bar{s})$ and $f_{\mp, v, h}(\bar{s})$ are CRDTs of the driven motion modified for the complex phase space representations for the driven motion:

$$
\begin{aligned}
& f_{\mp, h, v}(\bar{s})=\frac{1}{\sqrt{1-\lambda_{v}^{2}}}\left\{e^{ \pm i\left[\Psi_{y, v}\left(\bar{s}, \bar{s}_{v}\right)-\Psi_{y}\left(\bar{s}, \bar{s}_{v}\right)\right]} f_{\mp, h}(\bar{s})-\lambda_{v} e^{ \pm i\left[\Psi_{y, v}\left(\bar{s}, \bar{s}_{v}\right)+\Psi_{y}\left(\bar{s}, \bar{s}_{v}\right)\right]} f_{ \pm, h}(\bar{s})\right\} \\
& f_{\mp, v, h}(\bar{s})=\frac{1}{\sqrt{1-\lambda_{h}^{2}}}\left\{e^{-i\left[\Psi_{x, h}\left(\bar{s}, \bar{s}_{h}\right)-\Psi_{x}\left(\bar{s}, \bar{s}_{h}\right)\right]} f_{\mp, v}(\bar{s})-\lambda_{h} e^{-i\left[\Psi_{x, h}\left(\bar{s}_{,}, \bar{s}_{h}\right)+\Psi_{x}\left(\bar{s}, \bar{s}_{h}\right)\right]} f_{ \pm, v}^{*}(\bar{s})\right\} .
\end{aligned}
$$

The complex phase space positions of Equations (83) and (84) and, hence, $f_{\mp, h, v}(\bar{s})$ and $f_{\mp, v, h}(\bar{s})$ are what we observe with BPMs for the coupled driven motion.

\section{From CRDTs of Driven Motion to CRDTs of Free Motion}

In this section, we present an analytical procedure to determine the CRDTs of the free motion, $f_{\mp}(\bar{s})$, from measured turn-by-turn complex phase space positions of the driven motion, $\tilde{x}_{h}(n ; \bar{s})$ and $\tilde{y}_{v}(n ; \bar{s})$. Like the case of the free coupled motion, the modified CRDTs of the driven motion, $f_{\mp, h, v}(\bar{s})$ and $f_{\mp, v, h}(\bar{s})$, are determined from the Fourier components of $\tilde{x}_{h}(n ; \bar{s})$ and $\tilde{y}_{v}(n ; \bar{s})$. From Equations (43) and (44), the Fourier components corresponding to the leading order modes are

$$
\begin{aligned}
& \mathscr{X}_{h}\left(-\nu_{x, h} ; \bar{s}\right)=A_{x, h} \sqrt{\beta_{x, h}(\bar{s})} e^{-i \psi_{x, h}\left(\bar{s}, \bar{s}_{h}\right)-i \phi_{h}} \\
& \mathscr{Y}_{v}\left(-\nu_{y, v} ; \bar{s}\right)=A_{y, v} \sqrt{\beta_{y, v}(\bar{s})} e^{-i \psi_{y, v}\left(\bar{s}_{,} \bar{s}_{v}\right)-i \phi_{v}} .
\end{aligned}
$$

From Equations (83) and (84), the Fourier components corresponding to the first order modes are

$$
\begin{aligned}
\mathscr{X}_{h}\left(-\nu_{y, v} ; \bar{s}\right) & =2 i A_{y, v} f_{-, v, h}(\bar{s}) \sqrt{\beta_{x, h}(\bar{s})} e^{-i \psi_{y, v}\left(\bar{s}_{,} \bar{s}_{v}\right)-i \phi_{v}} \\
\mathscr{X}_{h}\left(\nu_{y, v} ; \bar{s}\right) & =2 i A_{y, v} f_{+, v, h}(\bar{s}) \sqrt{\beta_{x, h}(\bar{s})} e^{i \psi_{y, v}\left(\bar{s}, \bar{s}_{v}\right)+i \phi_{v}} \\
\mathscr{Y}_{v}\left(-\nu_{x, h} ; \bar{s}\right) & =2 i A_{x, h} f_{-, h, v}^{*}(\bar{s}) \sqrt{\beta_{y, v}(\bar{s})} e^{-i \psi_{x, h}\left(\bar{s}, \bar{s}_{h}\right)-i \phi_{h}} \\
\mathscr{Y}_{v}\left(\nu_{x, h} ; \bar{s}\right) & =2 i A_{x, h} f_{+, h, v}(\bar{s}) \sqrt{\beta_{y, v}(\bar{s})} e^{i \psi_{x, h}\left(\bar{s}, \bar{s}_{h}\right)+i \phi_{h}} .
\end{aligned}
$$


With these six components, $f_{\mp, h, v}(\bar{s})$ and $f_{\mp, v, h}(\bar{s})$ are determined by

$$
\begin{aligned}
& f_{-, h, v}(\bar{s})=\frac{1}{-2 i} \sqrt{\frac{\beta_{x, h}(\bar{s})}{\beta_{y, v}(\bar{s})}} \frac{\mathscr{Y}_{v}^{*}\left(-\nu_{x, h} ; \bar{s}\right)}{\mathscr{X}_{h}^{*}\left(-\nu_{x, h} ; \bar{s}\right)} \\
& f_{-, v, h}(\bar{s})=\frac{1}{2 i} \sqrt{\frac{\beta_{y, v}(\bar{s})}{\beta_{x, h}(\bar{s})}} \frac{\mathscr{X}_{h}\left(-\nu_{y, v} ; \bar{s}\right)}{\mathscr{Y}_{v}\left(-\nu_{y, v} ; \bar{s}\right)} \\
& f_{+, h, v}(\bar{s})=\frac{1}{2 i} \sqrt{\frac{\beta_{x, h}(\bar{s})}{\beta_{y, v}(\bar{s})}} \frac{\mathscr{Y}_{v}\left(\nu_{x, h} ; \bar{s}\right)}{\mathscr{X}_{h}^{*}\left(-\nu_{x, h} ; \bar{s}\right)} \\
& f_{+, v, h}(\bar{s})=\frac{1}{2 i} \sqrt{\frac{\beta_{y, v}(\bar{s})}{\beta_{x, h}(\bar{s})}} \frac{\mathscr{X}_{h}\left(\nu_{y, v} ; \bar{s}\right)}{\mathscr{Y}_{v}^{*}\left(-\nu_{y, v} ; \bar{s}\right)} .
\end{aligned}
$$

Once $f_{\mp, h, v}(\bar{s})$ and $f_{\mp, v, h}(\bar{s})$ are known, the CRDTs of the driven motion, $f_{\mp, h}(\bar{s})$ and $f_{\mp, v}(\bar{s})$, are determined by solving Equations $(85)$ and $(86)$ for $f_{\mp, h}(\bar{s})$ and $f_{\mp, v}(\bar{s})$ :

$$
\begin{aligned}
& f_{\mp, h}(\bar{s})=\frac{1}{\sqrt{1-\lambda_{v}^{2}}}\left\{e^{\mp i\left[\Psi_{y, v}\left(\bar{s}, \bar{s}_{v}\right)-\Psi_{y}\left(\bar{s}, \bar{s}_{v}\right)\right]} f_{\mp, h, v}(\bar{s})+\lambda_{v} e^{ \pm i\left[\Psi_{y, v}\left(\bar{s}, \bar{s}_{v}\right)+\Psi_{y}\left(\bar{s}_{\bar{s}}\right)\right]} f_{ \pm, h, v}(\bar{s})\right\} \\
& f_{\mp, v}(\bar{s})=\frac{1}{\sqrt{1-\lambda_{h}^{2}}}\left\{e^{i\left[\Psi_{x, h}\left(\bar{s}, \bar{s}_{h}\right)-\Psi_{x}\left(\bar{s}, \bar{s}_{h}\right)\right]} f_{\mp, v, h}(\bar{s})+\lambda_{h} e^{-i\left[\Psi_{x, h}\left(\bar{s}, \bar{s}_{h}\right)+\Psi_{x}\left(\bar{s}, \bar{s}_{h}\right)\right]} f_{ \pm, v, h}^{*}(\bar{s})\right\}
\end{aligned}
$$

We can determine the CRDTs of the free motion, $f_{\mp}(\bar{s})$, from those of the driven motion, $f_{\mp, h}(\bar{s})$ and $f_{\mp, v}(\bar{s})$, in the following way. If we exchange all the optical parameters of the horizontal plane for the free motion, $\beta_{x}(\bar{s}), \psi_{x}\left(\bar{s}_{2}, \bar{s}_{1}\right)$, $\Psi_{x}\left(\bar{s}_{2}, \bar{s}_{1}\right)$, and $\nu_{x}$, with those for the driven motion, $\beta_{x, h}(\bar{s}), \psi_{x, h}\left(\bar{s}_{2}, \bar{s}_{1}\right), \Psi_{x, h}\left(\bar{s}_{2}, \bar{s}_{1}\right)$, and $\nu_{x, h}$, in Equation $(77)$, we get

$$
\begin{aligned}
f_{\mp}(\bar{s})= & \frac{1}{\sqrt{1-\lambda_{h}^{2}}} \frac{\sin \left[\pi\left(\nu_{x, h} \mp \nu_{y}\right)\right]}{\sin \left[\pi\left(\nu_{x} \mp \nu_{y}\right)\right]}\left\{e^{i\left[\psi_{x, h}\left(\bar{s}_{,}, \bar{s}_{h}\right)-\psi_{x}\left(\bar{s}_{,}, \bar{s}_{h}\right)\right]} f_{\mp, h}(\bar{s})-\lambda_{h} e^{-i\left[\psi_{x, h}\left(\bar{s}, \bar{s}_{h}\right)+\psi_{x}\left(\bar{s}, \bar{s}_{h}\right)\right]}\left[\lambda_{c, h}^{\mp 1} f_{ \pm, h}^{*}(\bar{s})\right]\right. \\
& \left.-2 i \sin \left(\pi \delta_{h}\right) e^{i\left[\Psi_{x, h}\left(\bar{s}, \bar{s}_{h}\right)-\Psi_{x}\left(\bar{s}, \bar{s}_{h}\right)\right]} f_{\mp, h}\left(\bar{s} ; \bar{s}, \bar{s}_{h}\right)-2 i \sin \left(\pi \delta_{h}\right) e^{-i\left[\Psi_{x, h}\left(\bar{s}, \bar{s}_{h}\right)+\Psi_{x}\left(\bar{s}, \bar{s}_{h}\right)\right]}\left[\lambda_{c, h}^{\mp 1} f_{ \pm, h}^{*}\left(\bar{s} ; \bar{s}_{,}, \bar{s}_{h}\right)\right]\right\} .
\end{aligned}
$$

Similarly, if we exchange all the optical parameters of the vertical plane for the free motion, $\beta_{y}(\bar{s}), \psi_{y}\left(\bar{s}_{2}, \bar{s}_{1}\right), \Psi_{y}\left(\bar{s}_{2}, \bar{s}_{1}\right)$, and $\nu_{y}$, with those for the driven motion, $\beta_{y, v}(\bar{s}), \psi_{y, v}\left(\bar{s}_{2}, \bar{s}_{1}\right), \Psi_{y, v}\left(\bar{s}_{2}, \bar{s}_{1}\right)$, and $\nu_{y, v}$, in Equation (78), we get

$$
\begin{aligned}
f_{\mp}(\bar{s})= & \frac{1}{\sqrt{1-\lambda_{v}^{2}}} \frac{\sin \left[\pi\left(\nu_{x} \mp \nu_{y, v}\right)\right]}{\sin \left[\pi\left(\nu_{x} \mp \nu_{y}\right)\right]}\left\{e^{\mp i\left[\psi_{y, v}\left(\bar{s}, \bar{s}_{v}\right)-\psi_{y}\left(\bar{s}, \bar{s}_{v}\right)\right]} f_{\mp, v}(\bar{s})+\lambda_{v} e^{ \pm i\left[\psi_{y, v}\left(\bar{s}, \bar{s}_{v}\right)+\psi_{y}\left(\bar{s}, \bar{s}_{v}\right)\right]}\left[\lambda_{c, v}^{\mp 1} f_{ \pm, v}(\bar{s})\right]\right. \\
& \left. \pm 2 i \sin \left(\pi \delta_{v}\right) e^{\mp i\left[\Psi_{y, v}\left(\bar{s}, \bar{s}_{v}\right)-\Psi_{y}\left(\bar{s}, \bar{s}_{v}\right)\right]} f_{\mp, v}\left(\bar{s} ; \bar{s}, \bar{s}_{v}\right) \mp 2 i \sin \left(\pi \delta_{v}\right) e^{ \pm i\left[\Psi_{y, v}\left(\bar{s}, \bar{s}_{v}\right)+\Psi_{y}\left(\bar{s}_{,}, \bar{s}_{v}\right)\right]}\left[\lambda_{c, v}^{\mp 1} f_{ \pm, v}\left(\bar{s} ; \bar{s}, \bar{s}_{v}\right)\right]\right\}
\end{aligned}
$$

In these equations, $\lambda_{c, h}$ and $\lambda_{c, v}$ are parameters similar to $\lambda_{c}, \lambda_{h}$, and $\lambda_{v}$ :

$$
\begin{aligned}
& \lambda_{c, h}=\frac{\sin \left[\pi\left(\nu_{x, h}-\nu_{y}\right)\right]}{\sin \left[\pi\left(\nu_{x, h}+\nu_{y}\right)\right]} \\
& \lambda_{c, v}=\frac{\sin \left[\pi\left(\nu_{x}-\nu_{y, v}\right)\right]}{\sin \left[\pi\left(\nu_{x}+\nu_{y, v}\right)\right]}
\end{aligned}
$$

and, similar to $f_{\mp}\left(\bar{s} ; \bar{s}, \bar{s}_{\text {ac }}\right)$ in Equation $(79), f_{\mp, h}\left(\bar{s} ; \bar{s}, \bar{s}_{h}\right)$ and $f_{\mp, v}\left(\bar{s} ; \bar{s}, \bar{s}_{v}\right)$ describe contributions to $f_{\mp, h}(\bar{s})$ and $f_{\mp, v}(\bar{s})$ from the skew quadrupole fields located between $\bar{s}_{h}$ and $\bar{s}$ and between $\bar{s}_{v}$ and $\bar{s}$ :

$$
\begin{aligned}
& f_{\mp, h}\left(\bar{s} ; \bar{s}, \bar{s}_{h}\right)=\frac{1}{8 i \sin \left[\pi\left(\nu_{x, h} \mp \nu_{y}\right)\right]} \sum_{j=1}^{N} \Theta\left(\bar{s}_{j} ; \bar{s}, \bar{s}_{h}\right) \kappa_{j} \sqrt{\beta_{x, h}\left(\bar{s}_{j}\right) \beta_{y}\left(\bar{s}_{j}\right)} e^{-i\left[\Psi_{x, h}\left(\bar{s}, \bar{s}_{j}\right) \mp \Psi_{y}\left(\bar{s}, \bar{s}_{j}\right)\right]} \\
& f_{\mp, v}\left(\bar{s} ; \bar{s}, \bar{s}_{v}\right)=\frac{1}{8 i \sin \left[\pi\left(\nu_{x} \mp \nu_{y, v}\right)\right]} \sum_{j=1}^{N} \Theta\left(\bar{s}_{j} ; \bar{s}_{,}, \bar{s}_{v}\right) \kappa_{j} \sqrt{\beta_{x}\left(\bar{s}_{j}\right) \beta_{y, v}\left(\bar{s}_{j}\right)} e^{-i\left[\Psi_{x}\left(\bar{s}, \bar{s}_{j}\right) \mp \Psi_{y, v}\left(\bar{s}, \bar{s}_{j}\right)\right]} .
\end{aligned}
$$


By using Equations $(81)$ and $(82), f_{\mp, h}\left(\bar{s} ; \bar{s}, \bar{s}_{h}\right)$ and $f_{\mp, v}\left(\bar{s} ; \bar{s}, \bar{s}_{v}\right)$ can be expressed with $f_{\mp, h}(\bar{s}), f_{\mp, v}(\bar{s}), f_{\mp, h}\left(\bar{s}_{h}\right)$, and $f_{\mp, v}\left(\bar{s}_{v}\right)$ :

$$
\begin{aligned}
& f_{\mp, h}\left(\bar{s} ; \bar{s}, \bar{s}_{h}\right)=\frac{e^{\pi i\left(\nu_{x, h} \mp \nu_{y}\right) \operatorname{sgn}\left(\bar{s}-\bar{s}_{h}\right)}}{2 i \sin \left[\pi\left(\nu_{x, h} \mp \nu_{y}\right)\right]}\left\{f_{\mp, h}(\bar{s})-e^{-i\left[\psi_{x, h}\left(\bar{s}, \bar{s}_{h}\right) \mp \psi_{y}\left(\bar{s}, \bar{s}_{h}\right)\right]} f_{\mp, h}\left(\bar{s}_{h}\right)\right\} \\
& f_{\mp, v}\left(\bar{s} ; \bar{s}, \bar{s}_{v}\right)=\frac{e^{\pi i\left(\nu_{x} \mp \nu_{y, v}\right) \operatorname{sgn}\left(\bar{s}-\bar{s}_{v}\right)}}{2 i \sin \left[\pi\left(\nu_{x} \mp \nu_{y, v}\right)\right]}\left\{f_{\mp, v}(\bar{s})-e^{-i\left[\psi_{x}\left(\bar{s}, \bar{s}_{v}\right) \mp \psi_{y, v}\left(\bar{s}, \bar{s}_{v}\right)\right]} f_{\mp, v}\left(\bar{s}_{v}\right)\right\} .
\end{aligned}
$$

If there are BPMs close to the locations of the horizontal and vertical AC dipoles, $\bar{s}_{h}$ and $\bar{s}_{v}$, we can measure $f_{\mp, h}\left(\bar{s}_{h}\right)$ and $f_{\mp, v}\left(\bar{s}_{v}\right)$ and determine $f_{\mp, h}\left(\bar{s} ; \bar{s}, \bar{s}_{h}\right)$ and $f_{\mp, v}\left(\bar{s} ; \bar{s}, \bar{s}_{v}\right)$. In this way, the CRDTs of the free motion, $f_{\mp}(\bar{s})$, are determined by substituting the measured $f_{\mp, h}(\bar{s}), f_{\mp, v}(\bar{s}), f_{\mp, h}\left(\bar{s}_{h}\right)$, and $f_{\mp, v}\left(\bar{s}_{v}\right)$ into Equations (99), (100), (105), and (106). We may see that, from Equations (93), (94), (95), and $(96), f_{\mp}(\bar{s})$ determined from Equations (99) and (100) are inversely affected by calibration errors of the horizontal and vertical pick-ups of the considered dual-plane BPM. Therefore, when calculating the amplitude of $f_{\mp}(\bar{s})$, we should take the square average as we did in Equations (66) and (67).

\section{Errors When Approximating $f_{\mp}(\bar{s})$ with $f_{\mp, h}(\bar{s})$ or $f_{\mp, v}(\bar{s})$}

In the previous section, we presented the procedure to determine the CRDTs of the free motion from the CRDTs of the driven motion. However, as we may see in Equations (77) and (78), aside from the scaling factors $\left\{\sin \left[\pi\left(\nu_{x} \mp\right.\right.\right.$ $\left.\left.\left.\nu_{y}\right)\right] / \sin \left[\pi\left(\nu_{x, h} \mp \nu_{y}\right)\right]\right\}$ and $\left\{\sin \left[\pi\left(\nu_{x} \mp \nu_{y}\right)\right] / \sin \left[\pi\left(\nu_{x} \mp \nu_{y, v}\right)\right]\right\}$, the differences between the CRDTs of the free and driven motions are only on the order of $\lambda_{h}$ or $\lambda_{v}$. If the required accuracy of a measurement is not very high, such small differences may be simply ignored. In this section, we estimate the errors if we approximate $f_{\mp}(\bar{s})$ with $f_{\mp, h}(\bar{s})$ or $f_{\mp, v}(\bar{s})$ by simply ignoring the differences between $f_{\mp}(\bar{s})$ and $f_{\mp, h}(\bar{s})$ and between $f_{\mp}(\bar{s})$ and $f_{\mp, v}(\bar{s})$ after removing obvious differences.

\subsection{When $\left|f_{+}(\bar{s})\right| \simeq\left|\lambda_{c} f_{-}(\bar{s})\right|$}

We consider a case when the amplitudes of the difference and sum CRDTs satisfy $\left|f_{+}(\bar{s})\right| \simeq\left|\lambda_{c} f_{-}(\bar{s})\right|$. We also assume that there are randomly distributed many skew quadrupole fields in the ring. In Equations (77) and (78), the overall scaling factors, $\left\{1 / \sqrt{1-\lambda_{h}^{2}} \sin \left[\pi\left(\nu_{x} \mp \nu_{y}\right)\right] / \sin \left[\pi\left(\nu_{x, h} \mp \nu_{y}\right)\right]\right\}$ and $\left\{1 / \sqrt{1-\lambda_{h}^{2}} \sin \left[\pi\left(\nu_{x} \mp \nu_{y}\right)\right] / \sin \left[\pi\left(\nu_{x} \mp \nu_{y, v}\right)\right]\right\}$, and the overall phase factors, $e^{-i\left[\psi_{x, h}\left(\bar{s}, \bar{s}_{h}\right)-\psi_{x}\left(\bar{s}, \bar{s}_{h}\right)\right]}$ and $e^{ \pm i\left[\psi_{y, v}\left(\bar{s}, \bar{s}_{v}\right)-\psi_{y}\left(\bar{s}, \bar{s}_{v}\right)\right]}$, are known parameters and they should not be included in errors. We introduce new parameters, $\hat{f}_{\mp, h}(\bar{s})$ and $\hat{f}_{\mp, v}(\bar{s})$, from which these factors are removed:

$$
\begin{aligned}
\hat{f}_{\mp, h}(\bar{s}) & =\sqrt{1-\lambda_{h}^{2}} \frac{\sin \left[\pi\left(\nu_{x, h} \mp \nu_{y}\right)\right]}{\sin \left[\pi\left(\nu_{x} \mp \nu_{y}\right)\right]} e^{i\left[\psi_{x, h}\left(\bar{s}, \bar{s}_{h}\right)-\psi_{x}\left(\bar{s}, \bar{s}_{h}\right)\right]} f_{\mp, h}(\bar{s}) \\
& =f_{\mp}(\bar{s})+\lambda_{h} e^{-2 i \psi_{x}\left(\bar{s}, \bar{s}_{h}\right)}\left[\lambda_{c}^{\mp 1} f_{ \pm}^{*}(\bar{s})\right]+2 \pi i \delta_{h} f_{\mp}\left(\bar{s} ; \bar{s}_{,}, \bar{s}_{h}\right)+2 \pi i \delta_{h} e^{-2 i \Psi_{x}\left(\bar{s}_{,} \bar{s}_{h}\right)}\left[\lambda_{c}^{\mp 1} f_{ \pm}^{*}\left(\bar{s} ; \bar{s}, \bar{s}_{h}\right)\right]+\mathcal{O}\left(\lambda_{h}^{2}\right)
\end{aligned}
$$

and

$$
\begin{aligned}
\hat{f}_{\mp, v}(\bar{s}) & =\sqrt{1-\lambda_{v}^{2}} \frac{\sin \left[\pi\left(\nu_{x} \mp \nu_{y, v}\right)\right]}{\sin \left[\pi\left(\nu_{x} \mp \nu_{y}\right)\right]} e^{\mp i\left[\psi_{y, v}\left(\bar{s}, \bar{s}_{v}\right)-\psi_{y}\left(\bar{s}, \bar{s}_{v}\right)\right]} f_{\mp, v}(\bar{s}) \\
& =f_{\mp}(\bar{s})-\lambda_{v} e^{ \pm 2 i \psi_{y}\left(\bar{s}, \bar{s}_{v}\right)}\left[\lambda_{c}^{\mp 1} f_{ \pm}(\bar{s})\right] \mp 2 \pi i \delta_{v} f_{\mp}\left(\bar{s} ; \bar{s}_{,}, \bar{s}_{v}\right) \pm 2 \pi i \delta_{v} e^{ \pm 2 i \Psi_{y}\left(\bar{s}, \bar{s}_{v}\right)}\left[\lambda_{c}^{\mp 1} f_{ \pm}\left(\bar{s} ; \bar{s}, \bar{s}_{v}\right)\right]+\mathcal{O}\left(\lambda_{v}^{2}\right) .
\end{aligned}
$$

The difference between $f_{\mp}(\bar{s})$ and $\hat{f}_{\mp, h}(\bar{s})$ or between $f_{\mp}(\bar{s})$ and $\hat{f}_{\mp, v}(\bar{s})$ gives a systematic error when we do not use the solution of Section 4 . When a complex number $z$ changes by a small amount $\delta z$, changes in the amplitude and phase are given by

$$
\begin{aligned}
\frac{|z+\delta z|-|z|}{|z|} & =\frac{\operatorname{Re}\left[z^{*} \delta z\right]}{|z|^{2}}+\mathcal{O}\left(|\delta z|^{2}\right) \\
\arg (z+\delta z)-\arg (z) & =\frac{\operatorname{Im}\left[z^{*} \delta z\right]}{|z|^{2}}+\mathcal{O}\left(|\delta z|^{2}\right) .
\end{aligned}
$$


Applying these two formulae to Equations (107) and (108), the amplitudes of $\hat{f}_{\mp, h}(\bar{s})$ and $\hat{f}_{\mp, v}(\bar{s})$ satisfy

$$
\begin{aligned}
\frac{\left|\hat{f}_{\mp, h}(\bar{s})\right|-\left|f_{\mp}(\bar{s})\right|}{\left|f_{\mp}(\bar{s})\right|}= & \lambda_{h} \frac{\left|\lambda_{c}^{\mp 1} f_{ \pm}(\bar{s})\right|}{\left|f_{\mp}(\bar{s})\right|} \cos \left\{\arg \left[f_{\mp}(\bar{s})\right]+\arg \left[f_{ \pm}(\bar{s})\right]+2 \psi_{x}\left(\bar{s}_{,}, \bar{s}_{h}\right)\right\} \\
& +2 \pi \delta_{h} \frac{\left|f_{\mp}\left(\bar{s} ; \bar{s}, \bar{s}_{h}\right)\right|}{\left|f_{\mp}(\bar{s})\right|} \sin \left\{\arg \left[f_{\mp}(\bar{s})\right]-\arg \left[f_{\mp}\left(\bar{s} ; \bar{s}, \bar{s}_{h}\right)\right]\right\} \\
& +2 \pi \delta_{h} \frac{\left|\lambda_{c}^{\mp 1} f_{ \pm}\left(\bar{s} ; \bar{s}, \bar{s}_{h}\right)\right|}{\left|f_{\mp}(\bar{s})\right|} \sin \left\{\arg \left[f_{\mp}(\bar{s})\right]+\arg \left[f_{ \pm}\left(\bar{s} ; \bar{s}, \bar{s}_{h}\right)\right]+2 \Psi_{x}\left(\bar{s}_{,}, \bar{s}_{h}\right)\right\}+\mathcal{O}\left(\lambda_{h}^{2}\right)
\end{aligned}
$$

and

$$
\begin{aligned}
\frac{\left|\hat{f}_{\mp, v}(\bar{s})\right|-\left|f_{\mp}(\bar{s})\right|}{\left|f_{\mp}(\bar{s})\right|}= & -\lambda_{v} \frac{\left|\lambda_{c}^{\mp 1} f_{ \pm}(\bar{s})\right|}{\left|f_{\mp}(\bar{s})\right|} \cos \left\{\arg \left[f_{\mp}(\bar{s})\right]-\arg \left[f_{ \pm}(\bar{s})\right] \mp 2 \psi_{y}\left(\bar{s}_{,}, \bar{s}_{v}\right)\right\} \\
& \mp 2 \pi \delta_{v} \frac{\left|f_{\mp}\left(\bar{s} ; \bar{s}, \bar{s}_{v}\right)\right|}{\left|f_{\mp}(\bar{s})\right|} \sin \left\{\arg \left[f_{\mp}(\bar{s})\right]-\arg \left[f_{\mp}\left(\bar{s} ; \bar{s}, \bar{s}_{v}\right)\right]\right\} \\
& \pm 2 \pi \delta_{v} \frac{\left|\lambda_{c}^{\mp 1} f_{ \pm}\left(\bar{s} ; \bar{s}_{,}, \bar{s}_{v}\right)\right|}{\left|f_{\mp}(\bar{s})\right|} \sin \left\{\arg \left[f_{\mp}(\bar{s})\right]-\arg \left[f_{ \pm}\left(\bar{s} ; \bar{s}_{,} \bar{s}_{v}\right)\right] \mp 2 \Psi_{y}\left(\bar{s}, \bar{s}_{v}\right)\right\}+\mathcal{O}\left(\lambda_{v}^{2}\right)
\end{aligned}
$$

and the phases of $\hat{f}_{\mp, h}(\bar{s})$ and $\hat{f}_{\mp, v}(\bar{s})$ satisfy

$$
\begin{aligned}
\arg \left[\hat{f}_{\mp, h}(\bar{s})\right]-\arg \left[f_{\mp}(\bar{s})\right]= & -\lambda_{h} \frac{\left|\lambda_{c}^{\mp 1} f_{ \pm}(\bar{s})\right|}{\left|f_{\mp}(\bar{s})\right|} \sin \left\{\arg \left[f_{\mp}(\bar{s})\right]+\arg \left[f_{ \pm}(\bar{s})\right]+2 \psi_{x}\left(\bar{s}, \bar{s}_{h}\right)\right\} \\
& +2 \pi \delta_{h} \frac{\left|f_{\mp}\left(\bar{s} ; \bar{s}, \bar{s}_{h}\right)\right|}{\left|f_{\mp}(\bar{s})\right|} \cos \left\{\arg \left[f_{\mp}(\bar{s})\right]-\arg \left[f_{\mp}\left(\bar{s} ; \bar{s}, \bar{s}_{h}\right)\right]\right\} \\
& +2 \pi \delta_{h} \frac{\left|\lambda_{c}^{\mp 1} f_{ \pm}\left(\bar{s} ; \bar{s}_{h}\right)\right|}{\left|f_{\mp}(\bar{s})\right|} \cos \left\{\arg \left[f_{\mp}(\bar{s})\right]+\arg \left[f_{ \pm}\left(\bar{s} ; \bar{s}, \bar{s}_{h}\right)\right]+2 \Psi_{x}\left(\bar{s}, \bar{s}_{h}\right)\right\}+\mathcal{O}\left(\lambda_{h}^{2}\right)
\end{aligned}
$$

and

$$
\begin{aligned}
\arg \left[\hat{f}_{\mp, v}(\bar{s})\right]-\arg \left[f_{\mp}(\bar{s})\right]= & \lambda_{v} \frac{\left|\lambda_{c}^{\mp 1} f_{ \pm}(\bar{s})\right|}{\left|f_{\mp}(\bar{s})\right|} \sin \left\{\arg \left[f_{\mp}(\bar{s})\right]-\arg \left[f_{ \pm}(\bar{s})\right] \mp 2 \psi_{y}\left(\bar{s}, \bar{s}_{v}\right)\right\} \\
& \mp 2 \pi \delta_{v} \frac{\left|f_{\mp}\left(\bar{s} ; \bar{s}, \bar{s}_{v}\right)\right|}{\left|f_{\mp}(\bar{s})\right|} \cos \left\{\arg \left[f_{\mp}(\bar{s})\right]-\arg \left[f_{\mp}\left(\bar{s} ; \bar{s}_{,}, \bar{s}_{v}\right)\right]\right\} \\
& \pm 2 \pi \delta_{v} \frac{\left|\lambda_{c}^{\mp 1} f_{ \pm}\left(\bar{s} ; \bar{s}, \bar{s}_{v}\right)\right|}{\left|f_{\mp}(\bar{s})\right|} \cos \left\{\arg \left[f_{\mp}(\bar{s})\right]-\arg \left[f_{ \pm}\left(\bar{s} ; \bar{s}, \bar{s}_{v}\right)\right] \mp 2 \Psi_{y}\left(\bar{s}, \bar{s}_{v}\right)\right\}+\mathcal{O}\left(\lambda_{v}^{2}\right) .
\end{aligned}
$$

We note that there are six difference phases in these equations and they are all incoherent ${ }^{1}$. From the initial assumptions, the following approximation holds:

$$
\left\langle\frac{\left|\lambda_{c}^{\mp 1} f_{ \pm}(\bar{s})\right|}{\left|f_{\mp}(\bar{s})\right|}\right\rangle_{\mathrm{rms}} \simeq\left\langle\frac{\left|f_{\mp}\left(\bar{s} ; \bar{s}, \bar{s}_{h}\right)\right|}{\left|f_{\mp}(\bar{s})\right|}\right\rangle_{\mathrm{rms}} \simeq\left\langle\frac{\left|\lambda_{c}^{\mp 1} f_{ \pm}\left(\bar{s} ; \bar{s}, \bar{s}_{h}\right)\right|}{\left|f_{\mp}(\bar{s})\right|}\right\rangle_{\mathrm{rms}} \simeq\left\langle\frac{\left|f_{\mp}\left(\bar{s} ; \bar{s}, \bar{s}_{v}\right)\right|}{\left|f_{\mp}(\bar{s})\right|}\right\rangle_{\mathrm{rms}} \simeq\left\langle\frac{\left|\lambda_{c}^{\mp 1} f_{ \pm}\left(\bar{s} ; \bar{s}, \bar{s}_{v}\right)\right|}{\left|f_{\mp}(\bar{s})\right|}\right\rangle_{\mathrm{rms}} \simeq 1
$$

Then, under these assumptions, the systematic errors in amplitude and phase are estimated as

$$
\begin{aligned}
& \left\langle\frac{\left|\hat{f}_{\mp, h}(\bar{s})\right|-\left|f_{\mp}(\bar{s})\right|}{\left|f_{\mp}(\bar{s})\right|}\right\rangle_{\mathrm{rms}} \simeq\left\langle\arg \left[\hat{f}_{\mp, h}(\bar{s})\right]-\arg \left[\hat{f}_{\mp}(\bar{s})\right]\right\rangle_{\mathrm{rms}} \simeq 2 \pi \delta_{h} \sqrt{1+\frac{1}{8 \sin \left(2 \pi \nu_{x}\right)}} \\
& \left\langle\frac{\left|\hat{f}_{\mp, v}(\bar{s})\right|-\left|f_{\mp}(\bar{s})\right|}{\left|f_{\mp}(\bar{s})\right|}\right\rangle_{\mathrm{rms}} \simeq\left\langle\arg \left[\hat{f}_{\mp, v}(\bar{s})\right]-\arg \left[\hat{f}_{\mp}(\bar{s})\right]\right\rangle_{\mathrm{rms}} \simeq 2 \pi \delta_{v} \sqrt{1+\frac{1}{8 \sin \left(2 \pi \nu_{y}\right)}} .
\end{aligned}
$$

\footnotetext{
${ }^{1}$ An exception is that the phases in the second terms of Equations (111), (112), (113) and $(114),\left\{\arg \left[f_{\mp}(\bar{s})\right]-\arg \left[f_{\mp}\left(\bar{s} ; \bar{s}, \bar{s}_{h}\right)\right]\right\}$ and $\left\{\arg \left[f_{\mp}(\bar{s})\right]-\arg \left[f_{\mp}\left(\bar{s} ; \bar{s}, \bar{s}_{v}\right)\right]\right\}$, become identical when the horizontal and vertical AC dipoles are at the same location. In this condition, if we choose $\delta_{h}=\delta_{v}$ and take the mean of $\hat{f}_{-, h}(\bar{s})$ and $\hat{f}_{-, v}(\bar{s})$ to estimate $f_{-}(\bar{s})$, the contributions from these terms cancel out each and the systematic error can be reduced. Similarly, if we choose $\delta_{h}=-\delta_{v}$, the systematic error in $f_{+}(\bar{s})$ can be reduced.
} 


\section{$5.2 \quad$ When $\left|f_{+}(\bar{s})\right| \simeq\left|f_{-}(\bar{s})\right|$}

We make a comment on a case when the difference and sum resonances have similar strengths, $\left|f_{+}(\bar{s})\right| \simeq\left|f_{-}(\bar{s})\right|$. In such a case, from Equations (77) and (78), the leading order terms of $\hat{f}_{-, h}(\bar{s})$ and $\hat{f}_{-, v}(\bar{s})$ are given by

$$
\begin{aligned}
& \hat{f}_{-, h}(\bar{s})=f_{-}(\bar{s})+\frac{\lambda_{h}}{\lambda_{c}} e^{-2 i \psi_{x}\left(\bar{s}_{,} \bar{s}_{h}\right)} f_{+}^{*}(\bar{s})+\frac{2 \pi i \delta_{h}}{\lambda_{c}} e^{-2 i \Psi_{x}\left(\bar{s}, \bar{s}_{h}\right)} f_{+}^{*}\left(\bar{s} ; \bar{s}, \bar{s}_{h}\right)+\mathcal{O}\left(\lambda_{h}\right) \\
& \hat{f}_{-, v}(\bar{s})=f_{-}(\bar{s})-\frac{\lambda_{v}}{\lambda_{c}} e^{2 i \psi_{y}\left(\bar{s}, \bar{s}_{v}\right)} f_{+}(\bar{s})+\frac{2 \pi i \delta_{v}}{\lambda_{c}} e^{2 i \Psi_{y}\left(\bar{s}, \bar{s}_{v}\right)} f_{+}\left(\bar{s} ; \bar{s}, \bar{s}_{v}\right)+\mathcal{O}\left(\lambda_{v}\right)
\end{aligned}
$$

Because $\lambda_{h} / \lambda_{c}, \pi \delta_{h} / \lambda_{c}, \lambda_{v} / \lambda_{c}$, and $\pi \delta_{v} / \lambda_{c}$ are on the order one, $\hat{f}_{-, h}(\bar{s})$ and $\hat{f}_{-, v}(\bar{s})$ have contributions from $f_{+}(\bar{s})$, $f_{+}\left(\bar{s} ; \bar{s}, \bar{s}_{h}\right)$, and $f_{+}\left(\bar{s} ; \bar{s}, \bar{s}_{v}\right)$ on the leading order. Therefore, in this condition, estimating $f_{-}(\bar{s})$ with $\hat{f}_{-, h}(\bar{s})$ or $\hat{f}_{-, v}(\bar{s})$ is not very accurate. Again, from Equations (77) and (78), up to the first order of $\lambda_{h}$ and $\lambda_{v}, \hat{f}_{+, h}(\bar{s})$ and $\hat{f}_{+, v}(\bar{s})$ are given by

$$
\begin{aligned}
& \hat{f}_{+, h}(\bar{s})=f_{+}(\bar{s})+2 \pi i \delta_{h} f_{+}\left(\bar{s} ; \bar{s}, \bar{s}_{h}\right)+\mathcal{O}\left(\lambda_{h}^{2}\right) \\
& \hat{f}_{+, v}(\bar{s})=f_{+}(\bar{s})+2 \pi i \delta_{v} f_{+}\left(\bar{s} ; \bar{s}, \bar{s}_{v}\right)+\mathcal{O}\left(\lambda_{v}^{2}\right) .
\end{aligned}
$$

Hence, the approximation for $f_{+}(\bar{s})$ is still on the order of $\lambda_{h}$ or $\lambda_{v}$.

\section{3 $f_{\mp, h}(\bar{s})$ and $f_{\mp, v}(\bar{s})$ vs. $f_{\mp, h, v}(\bar{s})$ and $f_{\mp, v, h}(\bar{s})$}

In Section 3, we saw that the parameters directly determined from the spectra of the driven motion are the modified CRDT of the driven motion, $f_{\mp, h, v}(\bar{s})$ and $f_{\mp, v, h}(\bar{s})$, instead of the CRDT of the driven motion, $f_{\mp, h}(\bar{s})$ and $f_{\mp, v}(\bar{s})$. We can calculate $f_{\mp, h}(\bar{s})$ and $f_{\mp, v}(\bar{s})$ from $f_{\mp, h, v}(\bar{s})$ and $f_{\mp, v, h}(\bar{s})$ or vice versa with Equations (85), (86), (97), and (98). We make a comment to approximate $f_{\mp}(\bar{s})$ with $f_{\mp, h, v}(\bar{s})$ or $f_{\mp, v, h}(\bar{s})$. As Section 5.1, we consider $\left|f_{+}(\bar{s})\right| \simeq\left|\lambda_{c} f_{-}(\bar{s})\right|$. From Equations (85) and (86), up to the first order of $\lambda_{c}, \lambda_{h}$, and $\lambda_{v}, f_{-, h, v}(\bar{s})$ and $f_{+, v, h}(\bar{s})$ are given by

$$
\begin{aligned}
& f_{-, h, v}(\bar{s})=e^{i\left[\Psi_{y, v}\left(\bar{s}, \bar{s}_{v}\right)-\Psi_{y}\left(\bar{s}, \bar{s}_{v}\right)\right]} f_{-, h}(\bar{s})+\mathcal{O}\left(\lambda_{v}^{2}, \lambda_{c} \lambda_{v}\right) \\
& f_{-, v, h}(\bar{s})=e^{-i\left[\Psi_{x, h}\left(\bar{s}, \bar{s}_{h}\right)-\Psi_{x}\left(\bar{s}, \bar{s}_{h}\right)\right]} f_{-, v}(\bar{s})+\mathcal{O}\left(\lambda_{v}^{2}, \lambda_{c} \lambda_{v}\right) .
\end{aligned}
$$

The differences between $f_{-, h, v}(\bar{s})$ and $f_{-, h}(\bar{s})$ and between $f_{+, h, v}(\bar{s})$ and $f_{+, h}(\bar{s})$ are only in the phases and on the orders of $\lambda_{v}$ and $\lambda_{h}$. Hence, when we approximate $f_{-}(\bar{s})$ with $f_{-, h, v}(\bar{s})$ or $f_{-, v, h}(\bar{s})$, we only have additional phase errors on the order $\lambda_{h}$ or $\lambda_{v}$, compared to when approximating with $f_{-, h}(\bar{s})$ or $f_{-, v}(\bar{s})$. Again from Equations (85) and (86), the leading order terms of $f_{+, h, v}(\bar{s})$ and $f_{+, v, h}(\bar{s})$ are given by

$$
\begin{aligned}
& f_{+, h, v}(\bar{s})=f_{+, h}(\bar{s})-\frac{\lambda_{v}}{\lambda_{c}} e^{-2 i \Psi_{y}\left(\bar{s}, \bar{s}_{v}\right)}\left[\lambda_{c} f_{-, h}(\bar{s})\right]+\mathcal{O}\left(\lambda_{v}\right) \\
& f_{+, v, h}(\bar{s})=f_{+, v}(\bar{s})-\frac{\lambda_{h}}{\lambda_{c}} e^{-2 i \Psi_{x}\left(\bar{s}, \bar{s}_{h}\right)}\left[\lambda_{c} f_{-, v}^{*}(\bar{s})\right]+\mathcal{O}\left(\lambda_{h}\right) .
\end{aligned}
$$

Because $\lambda_{v} / \lambda_{c}$ and $\lambda_{h} / \lambda_{c}$ are on the order of one, $f_{+, h, v}(\bar{s})$ and $f_{+, v, h}(\bar{s})$ have contributions from $f_{-, h}(\bar{s})$ and $f_{-, v}(\bar{s})$ on the leading order. Hence, the approximation of the sum CRDT $f_{+}(\bar{s})$ with $f_{+, h, v}(\bar{s})$ or $f_{+, v, h}(\bar{s})$ may not be very accurate.

\section{Conclusions}

This note derived an expression of the coupled driven betatron motion, showed that it is parametrized with a new set of CRDTs, similar to the case of the uncoupled driven motion, and presented how to extrapolate the CRDTs of the free motion from those of the driven motion, which are directly determined from spectra of the driven motion. Please note that the presented derivation and parametrization of the coupled driven motion with a new set of CRDTs can be extended in a straight forward manner to the driven motion under influences of any perturbative fields characterized by any resonance driving terms. We also gave estimates of errors when we approximate the CRDTs of the free motion with those of the driven motion. Once we removed the trivial differences in amplitude and phase, accuracy of the approximation is on the order of $2 \pi \delta_{h}$ or $2 \pi \delta_{v}$ in typical conditions, where magnitude of the sum resonance is smaller than that of the difference resonance by the order of $\lambda_{c}$. This note only presented the analytic solution and estimate of the problem. The obvious next step is to perform numerical simulations and develop an algorithm for real data. 


\section{Acknowledgments}

The Author would like to thank to G. Vanbavinckhove and R. Tomás for useful discussions and encouragement to write this note.

\section{References}

[1] M. Bai, S. Y. Lee, J. W. Glenn, H. Huang, L. Ratner, T. Roser, M. J. Syphers, and W. van Asselt, "Experimental Test of Coherent Betatron Resonance Excitations", Phys. Rev. E 56, p. 6002 (1997).

[2] R. Bartolini and F. Schmidt, "Normal Form via Tracking or Beam Data", Part. Accel. 59, p. 93 (1998).

[3] S. Peggs, "Nonlinear Diagnostics Using AC Dipoles", in Proceedings of the 18th Particle Accelerator Conference, New York, New York, 1999 (IEEE, Piscataway, NJ, 1999), p. 1572.

[4] R. Tomás, "Adiabaticity of the Ramping Process of an ac Dipole", Phys. Rev. ST Accel. Beams 8, 024401 (2005).

[5] R. Miyamoto, S. E. Kopp, A. Jansson, and M. J. Syphers "Parametrization of the Driven Betatron Oscillation", Phys. Rev. ST Accel. Beams 11, 084002 (2008).

[6] R. Tomás, "Normal form of particle motion under the influence of an ac dipole", Phys. Rev. ST Accel. Beams 5, 054001 (2002).

[7] R. Calaga, R. Tomás, and A. Franchi, "Global and Local Coupling Compensation in RHIC Using AC Dipoles"

[8] E. D. Courant and H. S. Snyder, "Theory of the Alternating-Gradient Synchrotron", Ann. Phys. 3, p. 1 (1958).

[9] D. D. Caussyn, M. Ball, B. Brabson, J. Collins, S. A. Curtis, V. Derenchuck, D. DuPlantis, G. East, M. Ellison, T. Ellison, D. Friesel, B. Hamilton, W. P. Jones, W. Lamble, S. Y. Lee, D. Li, M. G. Minty, T. Sloan, and G. Xu, "Experimental studies of nonlinear beam dynamics", Phys. Rev. A 46, p. 7942 (1992).

[10] M. Hayes, F. Schmidt, and R. Tomás, "Direct Measurement of Resonance Driving Terms at SPS at 26 GeV", in Proceedings of the 8th European Particle Accelerator Conference, Paris, France, 2002 (EPS-IGA and CERN, Geneva, 2002), p. 1290.

[11] R. Tomás, M. Bai, R. Calaga, and W. Fischer, "Measurement of Global and Local Resonance Terms", Phys. Rev. ST Accel. Beams 8, 024001 (2005). 